\title{
Síndrome Metabólica na Doença Arterial Coronariana e Vascular Oclusiva: Uma Revisão Sistemática
}

\author{
Metabolic Syndrome in Coronary Artery and Occlusive Vascular Diseases: A Systematic Review \\ Daniela Reis Elbert Farias, Avany Fernandes Pereira, Glorimar Rosa \\ Instituto de Nutrição Josué de Castro - Universidade Federal do Rio de Janeiro, Rio de Janeiro, RJ, Brasil
}

\section{Resumo}

Atualmente, a síndrome metabólica (SM) se mostra altamente prevalente, sendo associada a fatores de risco para doenças crônicas não transmissíveis, tais como diabetes mellitus tipo 2, doenças ateroscleróticas e coronarianas. O objetivo desta revisão sistemática foi descrever os resultados de estudos que investigaram a associação da SM com a doença arterial coronariana e doenças vasculares oclusivas. Foi realizada a revisão sistemática com dados de estudos originais publicados entre 1999 e 2008, escritos em inglês ou português, utilizando-se as bases de dados Medline, Pubmed, Highwire Press e Science Direct. Foram incluídos artigos que fizeram o diagnóstico da SM através do critério do National Cholesterol Education Program - Adult Treatment Panel III (NCEP ATP III, 2001). Foram excluídos estudos realizados com animais, de suplementação e que realizaram administração oral ou endovenosa de qualquer substância, assim como aqueles de baixa qualidade metodológica e com amostra inicialmente heterogênea. Apesar da heterogeneidade entre os estudos, observou-se que indivíduos com SM apresentam maior probabilidade (risco $=2,13$ ) de desenvolverem as doenças vasculares oclusivas, doença coronariana, diabetes mellitus e acidente vascular encefálico. Mudanças no estilo de vida, como práticas alimentares saudáveis, atividade física regular e a cessação do tabagismo devem ser incentivadas pelos profissionais da saúde a fim de minimizar as complicações e a morbimortalidade associada à SM.

\section{Introdução}

A síndrome metabólica (SM) é caracterizada por alterações metabólicas relacionadas à obesidade abdominal e resistência à insulina. De acordo com o NCEP ATP III $^{1}$, seu diagnóstico é realizado pela ocorrência de três ou mais das seguintes condições: obesidade abdominal (circunferência da cintura acima de $102 \mathrm{~cm}$ no sexo masculino e $88 \mathrm{~cm}$ no sexo feminino); hipertrigliceridemia (superior ou igual a $150 \mathrm{mg} / \mathrm{dl}$ ); baixas concentrações de HDL-colesterol (menor que 40mg/dl no sexo masculino e menor que $50 \mathrm{mg} / \mathrm{dl}$ no sexo feminino);

\section{Palavras chave}

Síndrome metabólica, doença da artéria coronariana, doenças vasculares, metanálise.

Correspondência: Daniela Reis Elbert Farias •

Rua Aureliano Coutinho, 88/303 - 25625-000 - Centro - Petrópolis, RJ - Brasil E-mail: elbert@compuland.com.br

Artigo recebido em 09/10/08; revisado recebido em 18/02/09; aceito em 30/04/09 pressão arterial sistólica acima ou igual a 130 mmHg e pressão arterial diastólica acima de $85 \mathrm{mmHg}$; e hiperglicemia de jejum (superior ou igual a $110 \mathrm{mg} / \mathrm{dl}$ ).

Existem outros critérios propostos para seu diagnóstico: o World Health Organization ${ }^{2}$ (WHO), que utiliza valores de microalbuminúria e resistência à insulina, o European Group for the Study of Insulin Resistance ${ }^{3}$ (EGIR), que utiliza a resistência à insulina como fator de risco obrigatório associado a dois ou mais fatores de risco; o American Heart Association/National Heart, Lung and Blood Institute 4 (AHA/NHLBI), que tem como critério a presença de três ou mais fatores de risco associados à doença arterial coronariana (DAC), e o critério da International Diabetes Federation ${ }^{5}$ (IDF), que utiliza valores diferenciados para a circunferência da cintura de acordo com a etnia. Apesar de muitas semelhanças entre os critérios, observa-se que o critério do NCEP ATP III ${ }^{1}$ é o mais utilizado na literatura científica ${ }^{4}$.

A prevalência mundial da SM é elevada, entre 20\% e 25\%, e bastante variável de acordo com o critério diagnóstico utilizado ${ }^{6-9}$. Independente do diagnóstico utilizado, sua prevalência é elevada em indivíduos com doenças cardiovasculares $(\mathrm{DCV})^{10}$. Não existem estudos epidemiológicos brasileiros que definam sua prevalência, no entanto, em estudo regional com 530 nipo-brasileiros, evidenciou-se uma prevalência de $21 \%{ }^{11}$, enquanto em um estudo realizado na população de migrantes espanhóis e seus descendentes no Brasil, foi observada uma prevalência de $35,6 \%{ }^{12}$.

A SM é considerada um fator de risco tão importante quanto o tabagismo para o desenvolvimento de doenças vasculares oclusivas e ateroscleróticas ${ }^{1,9,13}$. Por isso, o diagnóstico e tratamento da SM são de extrema importância já que sua prevalência é crescente em todo o mundo.

\section{Objetivo}

Descrever os resultados de estudos clínicos que investigaram o impacto da SM, diagnosticada pelo critério do NCEP ATP III ${ }^{1}$, na ocorrência da DAC e doenças vasculares oclusivas em indivíduos com idade superior a 18 anos.

\section{Métodos}

O levantamento bibliográfico foi realizado em 2008, sendo consultadas as seguintes bases de dados eletrônicas: Medline, Scielo, Pubmed, Highwire Press e Science Direct.. A busca retrospectiva se limitou aos artigos científicos indexados, originais como estudos clínicos, randomizados e não randomizados, transversais, prospectivos, de coorte e de base populacional, que envolveram seres humanos com idade 


\section{Atualização Clínica}

igual ou superior a 18 anos e que foram publicados entre 2004 e 2008, escritos nas línguas inglesa ou portuguesa, tendo sido utilizadas a combinação das seguintes palavras-chave: síndrome metabólica e doença arterial coronariana; síndrome metabólica e doenças vasculares. Outra estratégia utilizada foi a seleção manual de referências bibliográficas em artigos encontrados.

Os artigos, identificados inicialmente por três pesquisadores diferentes, foram selecionados pela estratégia de busca inicial a partir dos seguintes critérios de elegibilidade: (1) artigos publicados nos últimos quatro anos; (2) artigos escritos nas línguas inglesa ou portuguesa; (3) estudos clínicos observacionais; (4) estudos randomizados ou não randomizados; (5) estudos realizados com seres humanos adultos ou idosos com SM; (6) diagnóstico clínico de doença arterial coronariana e/ou de doenças vasculares oclusivas; e (7) estudos apresentando como desfecho principal a associação das anormalidades metabólicas da síndrome com a presença dessas doenças. Foram incluídos artigos que utilizaram o critério do NCEP ATP III ${ }^{1}$ para o diagnóstico da SM e aqueles que utilizaram juntamente com o NCEP ATP III ${ }^{2}$ outro critério para o diagnóstico da $\mathrm{SM}$, tais como o $\mathrm{WHO}^{2}, \mathrm{EGIR}^{3}, \mathrm{AHA} /$ $\mathrm{NHLBI}^{4}$, IDF ${ }^{5}$, e American College of Endocrinology (ACE) ${ }^{14}$. Foram excluídos, de acordo com critérios definidos antes da busca, os estudos realizados com animais, que fizeram suplementação e que realizaram administração oral ou endovenosa de qualquer substância, assim como aqueles de baixa qualidade metodológica, como amostra heterogênea, ou informações disponíveis em artigos mais recentes.

Os estudos selecionados tiveram como desfecho principal a associação das anormalidades metabólicas dos indivíduos com SM com a presença de doença arterial coronariana e/ ou doenças vasculares oclusivas ${ }^{15}$. A qualidade metodológica dos estudos foi avaliada considerando alguns aspectos, tais como: especificação dos critérios de inclusão e exclusão do estudo; alocação aleatória dos participantes do estudo; similaridade entre os grupos de estudo e controle na fase inicial do estudo; e relato da análise estatística empregada. Finalmente, tais artigos foram revisados para evitar inclusão de dados em duplicata.

\section{Análise dos dados}

Os dados foram analisados pelo programa RevMan version 5,0 (The Cochrane Collaboration Copenhagen) ${ }^{16}$. Para avaliar a heterogeneidade entre os estudos, realizou-se o teste $\mathrm{Chi}^{2}$, considerando como graus de liberdade $\mathrm{n}-1$, onde " $\mathrm{n}$ " é igual ao número de estudos. Calculou-se a heterogeneidade significativa utilizando o modelo de efeito fixo. A inconsistência $\left(\mathrm{I}^{2}\right)$ foi calculada para verificar a extensão da diferença entre os estudos que contemplavam grupos com e sem SM que apresentavam DAC ou doenças vasculares oclusivas, sendo considerada baixa $25 \%$, moderada $50 \%$ e alta maior que $75 \%{ }^{17}$.

\section{Resultados}

Foram identificados 42 artigos, dos quais 30 utilizaram o critério do NCEP ATP III (2001) ${ }^{1}$ como único critério diagnóstico da SM, e 12 artigos utilizaram outros critérios para o diagnóstico da SM além do NCEP ATP III ${ }^{1}$. Foram selecionados artigos, sendo: 14 do tipo coorte ${ }^{13,15-29}$,
12 transversais ${ }^{10,30-40}, 8$ de coorte prospectivos ${ }^{41-48}, 2$ observacionais $^{49,50}, 2$ prospectivos ${ }^{51,52}$, e 1 para cada tipo de estudo: prospectivo e observacional ${ }^{53}$, transversal e de coorte populacional ${ }^{54}$, caso-controle ${ }^{55}$ e longitudinal de base comunitária ${ }^{56}$.

O maior número de publicações foi proveniente dos Estados Unidos ( $\mathrm{n}=17$ ), dos quais 12 artigos utilizaram apenas o critério NCEP ATP III (2001), seguidos da Itália $(n=6)$ e Holanda $(n=3)$. Dois artigos foram selecionados em cada um dos seguintes países: Inglaterra, Grécia, França, Canadá, Finlândia e Turquia; e nos demais países, Japão, Argentina, Noruega, e Áustria, apenas 1 artigo foi selecionado.

As características metodológicas desses estudos, além do ano de publicação, país de origem, população estudada, grupo de estudo, faixa etária, critério utilizado para o diagnóstico da SM, tipo e duração do estudo e resultados principais, foram apresentados na tabela 1.

Alguns estudos não mencionaram o tempo de duração do estudo. Entre os estudos nos quais esses dados foram obtidos foi possível observar que não houve duração inferior a 6 meses $^{28}$, tendo sido o maior tempo de seguimento de 20 anos $^{53}$.

A forma de recrutamento dos participantes foi especificada em todos os estudos. O tamanho amostral variou de 83 a 15.922 indivíduos, sendo que dos 42 estudos selecionados, apenas 2 apresentaram amostra total inferior a 100 indivíduos, sendo de 83 e 87 indivíduos cada um desses estudos ${ }^{32,37}$.

A maioria dos estudos incluiu indivíduos de ambos os sexos ( $n=36)$, com a exceção de 5 estudos que incluíram apenas mulheres ${ }^{28,34,37,48,50}$ e 1 estudo que incluiu apenas homens na amostra ${ }^{43}$. Dos estudos selecionados, 50\% não mencionaram a faixa etária da amostra, no entanto a média e o desvio padrão da idade dos grupos estudados foram mencionadas em 31 (73,8\%) artigos selecionados. Dos artigos que não mencionaram o desvio padrão da idade dos grupos estudados, 3 mencionaram apenas a média de idade dos grupos, 3 apenas a faixa etária dos grupos e 5 a média de idade e a faixa etária dos grupos.

\section{Discussão}

A SM apresenta-se evidenciada devido a sua elevada prevalência e por representar um importante conjunto de fatores de risco cardiovascular, frequentemente associados à deposição de gordura central e à resistência à insulina ${ }^{57}$. Do ponto de vista epidemiológico, a SM é responsável pelo aumento estimado da mortalidade geral em 1,5 vez, e por DAC e doenças vasculares oclusivas em 2,5 vezes ${ }^{58}$. Apesar de sua relevância, no Brasil ainda existem carências de dados sobre suas características epidemiológicas e de estudos que verifiquem a sua relação com o aumento da morbimortalidade por doenças vasculares oclusivas e DAC. O estudo da SM também tem sido dificultado pela ausência de consenso no critério para o seu diagnóstico e nos pontos de corte dos seus fatores de risco, causando relevantes repercussões na prática clínica e na saúde pública. Nesta revisão, optamos pela seleção de artigos que utilizaram o critério do NCEP ATP III ${ }^{1}$ para o diagnóstico da SM, não só por esse ser um critério que apresenta fácil aplicabilidade na prática clínica, 


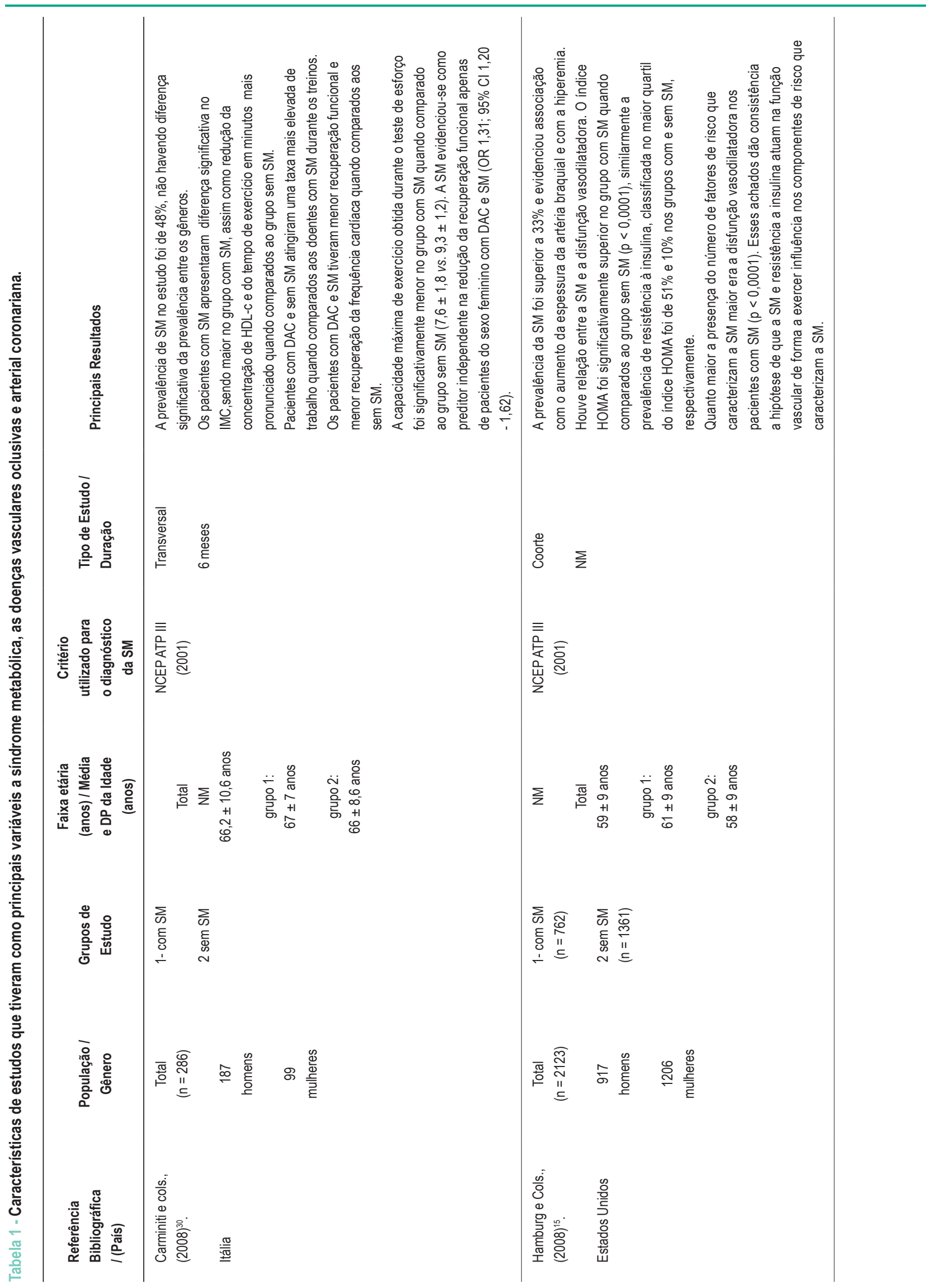




\section{Atualização Clínica}

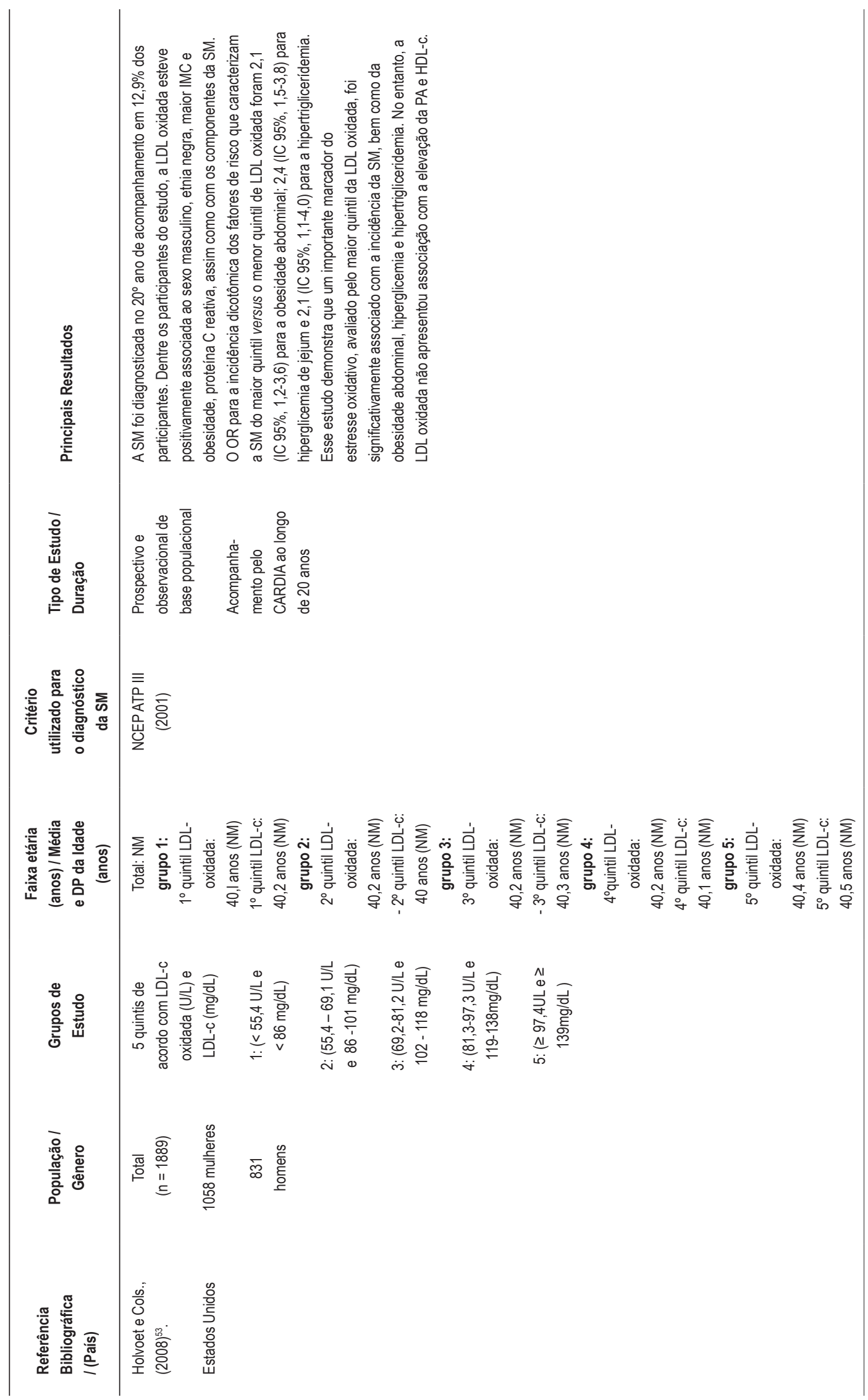




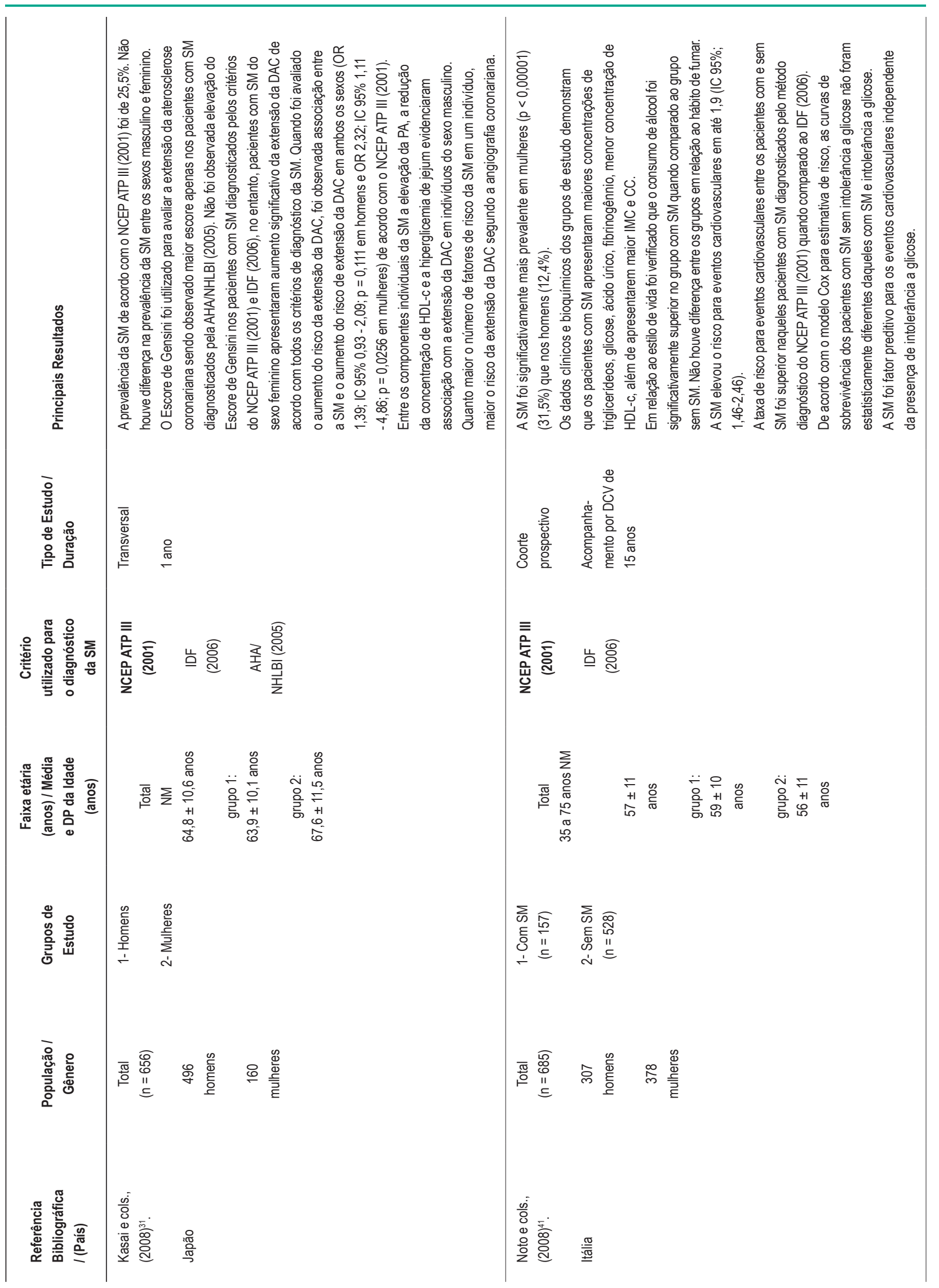




\section{Atualização Clínica}

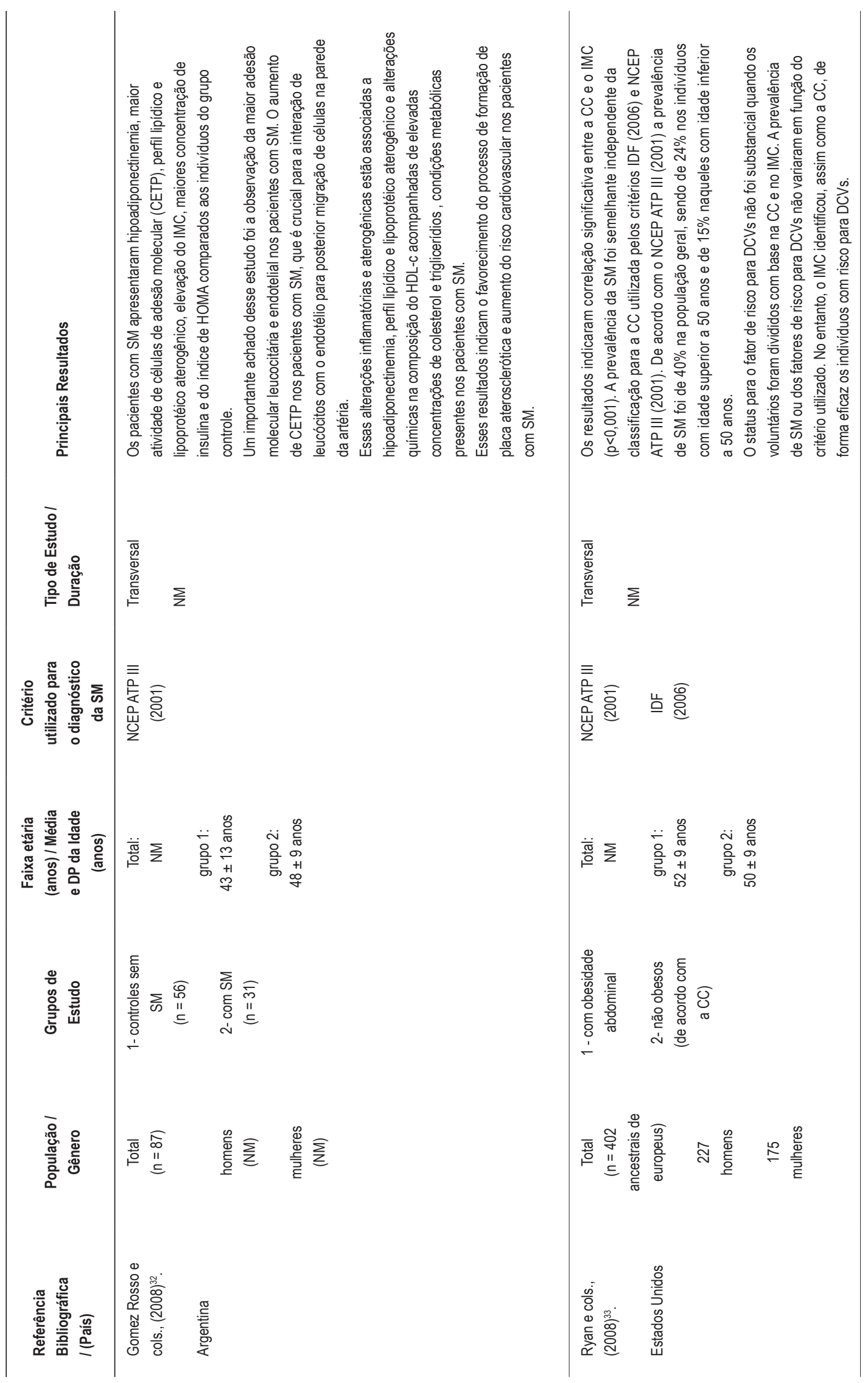




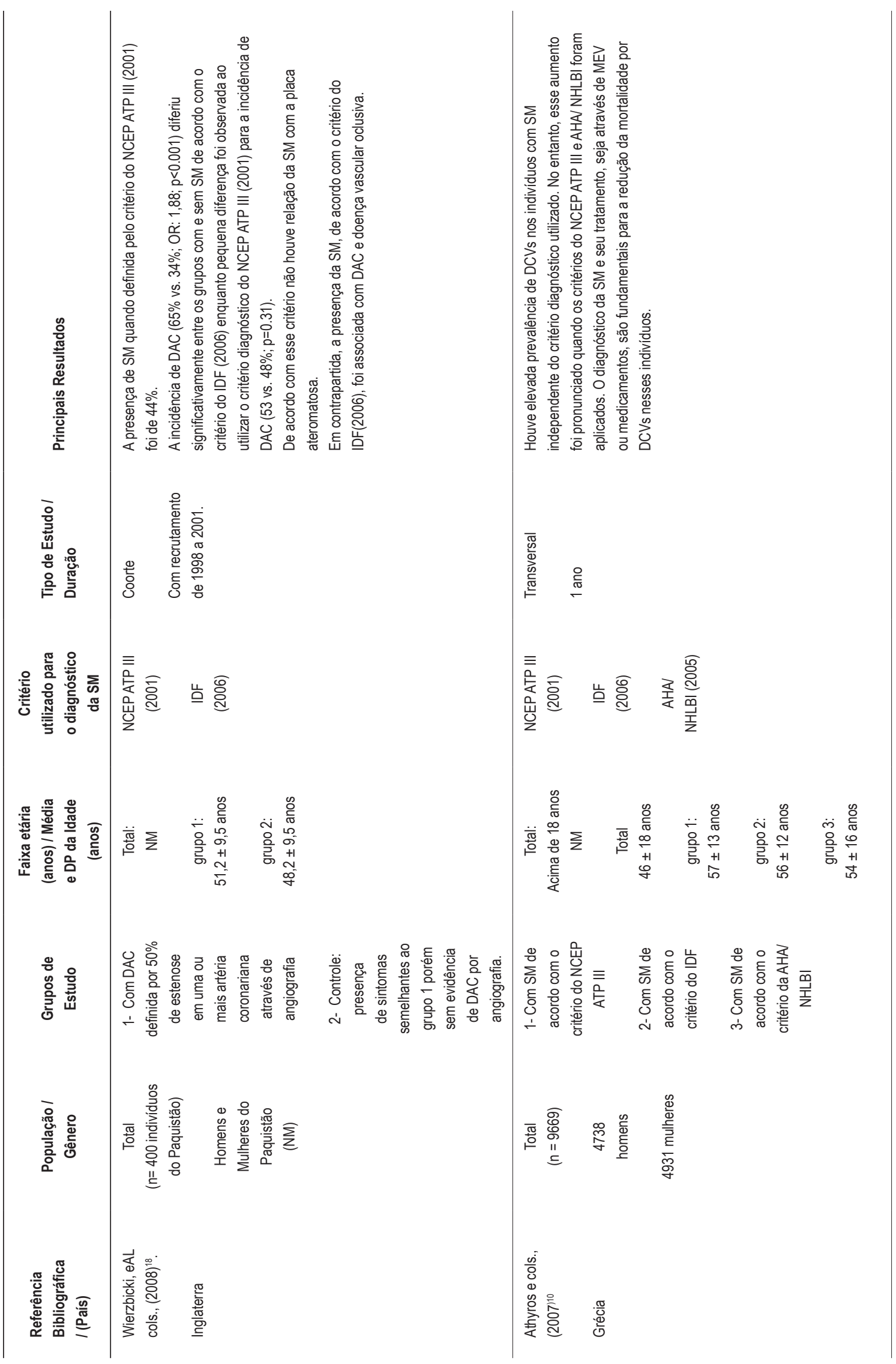




\section{Atualização Clínica}

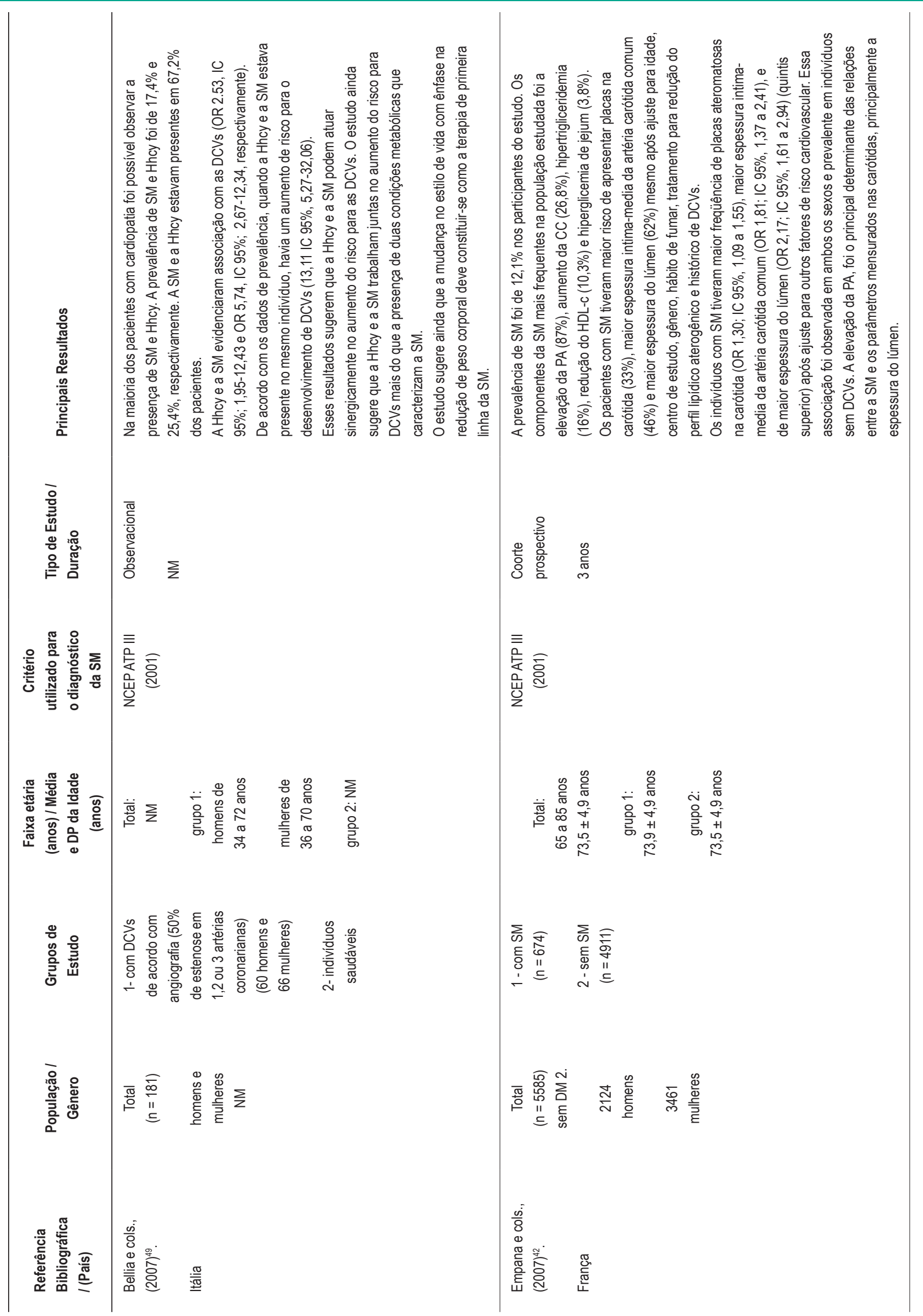




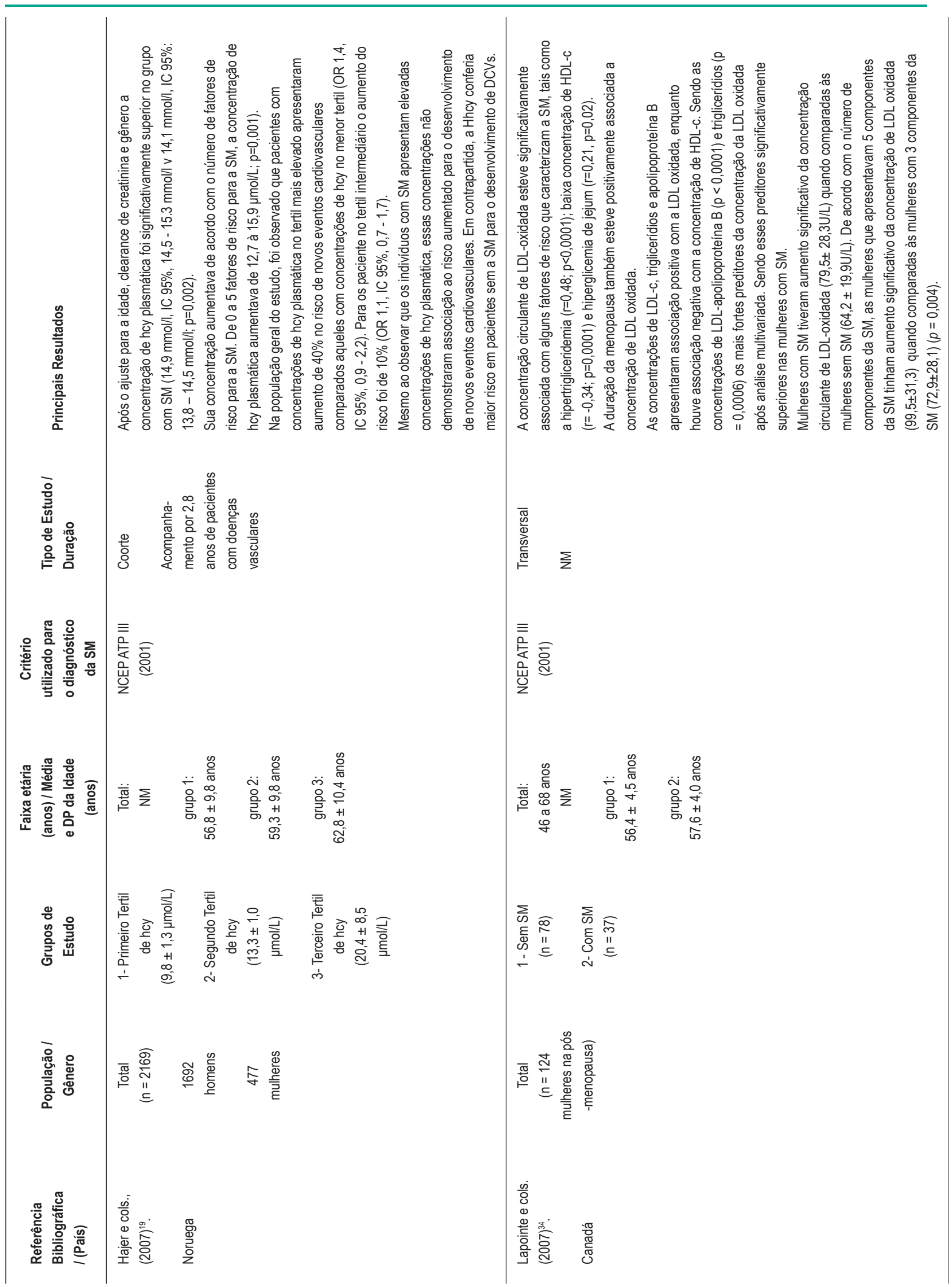




\section{Atualização Clínica}

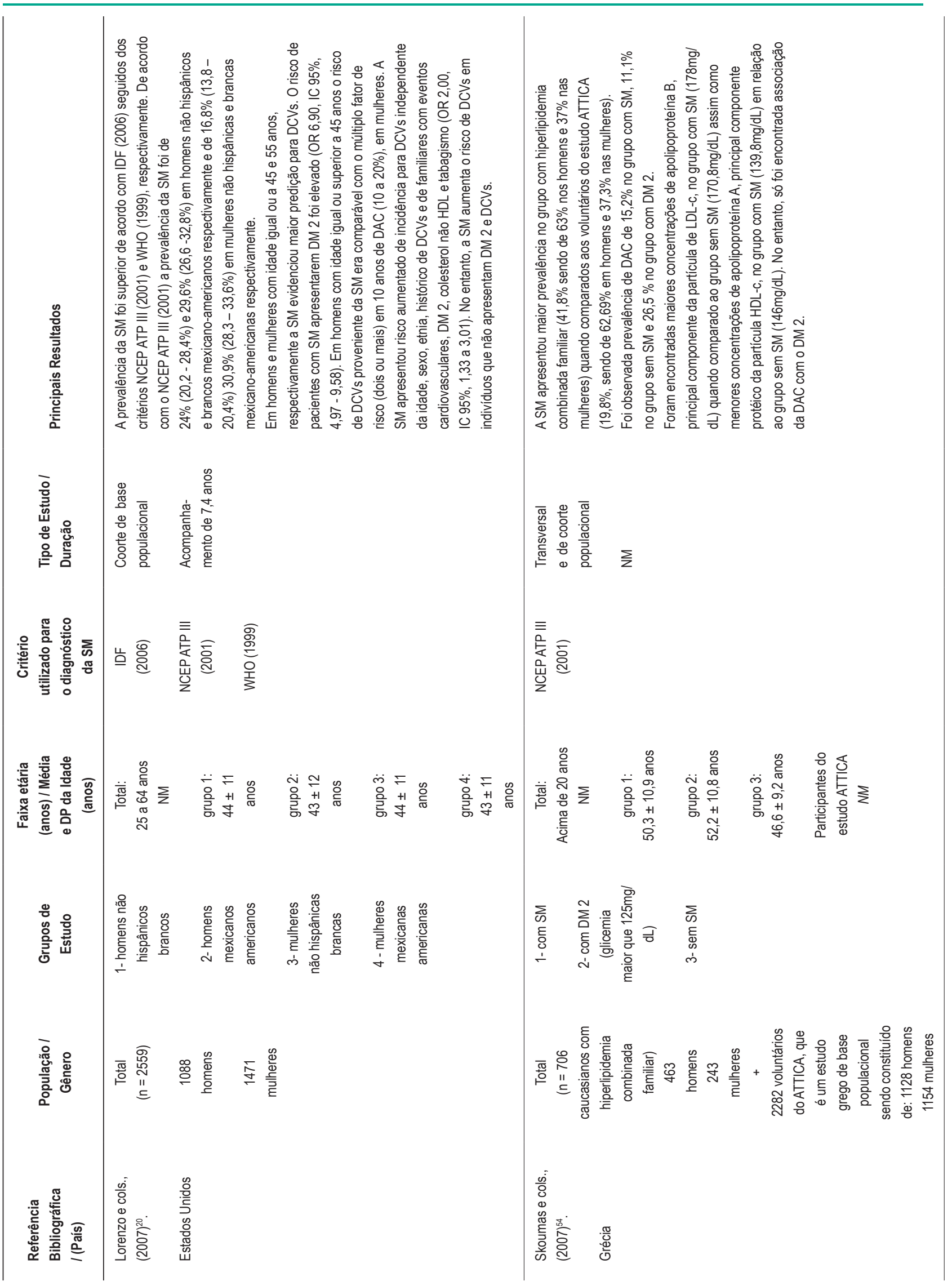




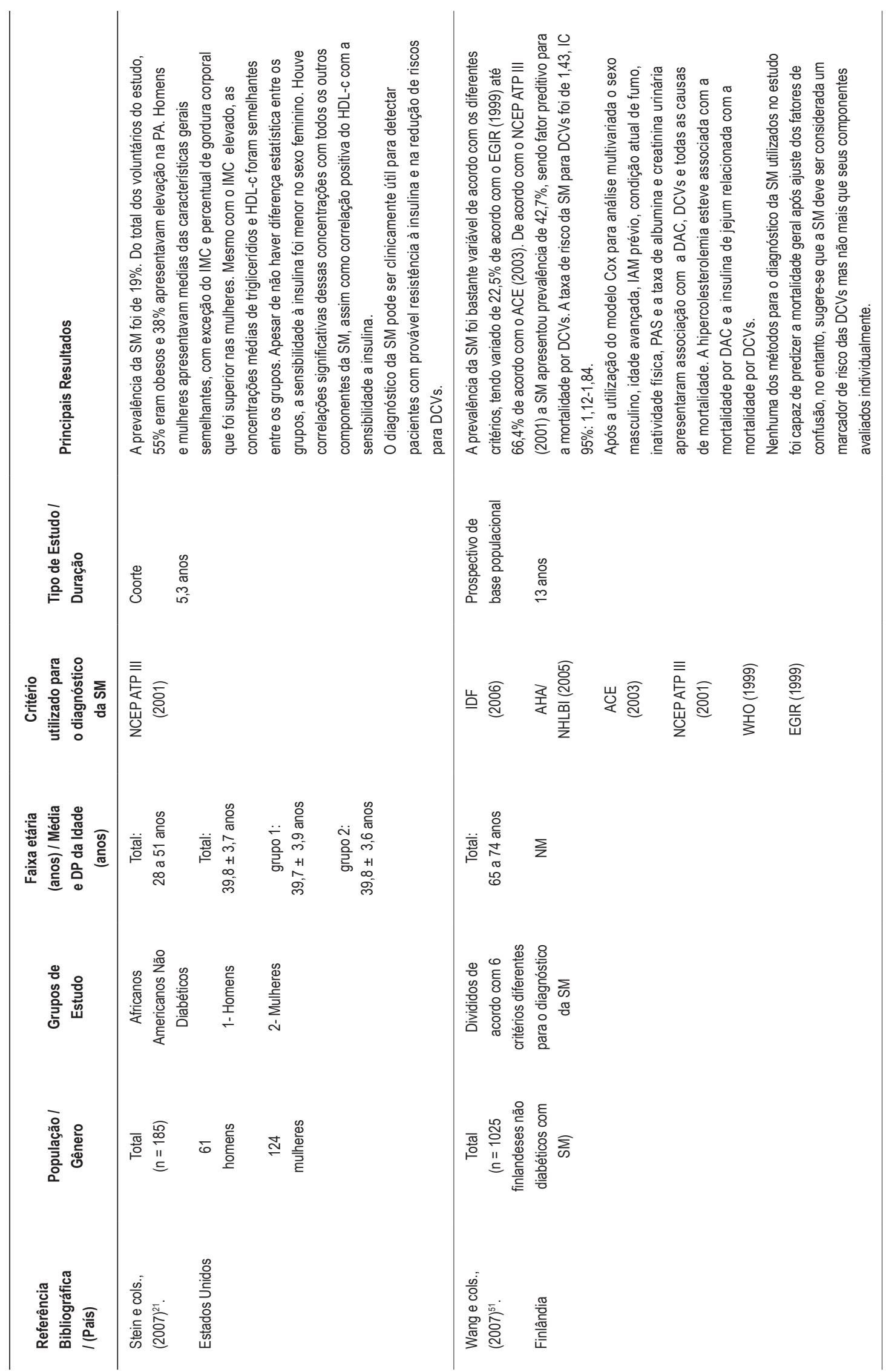




\section{Atualização Clínica}

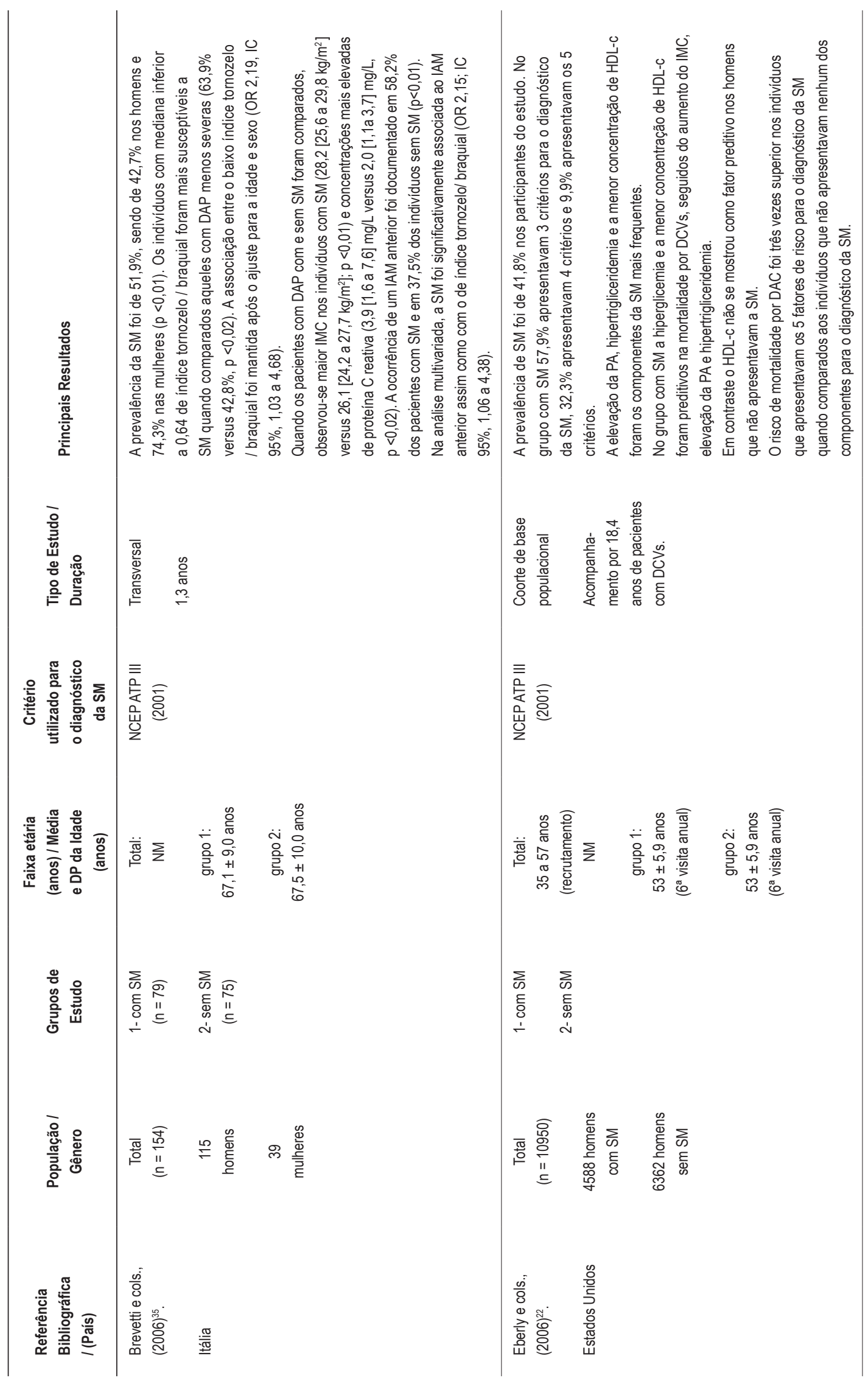




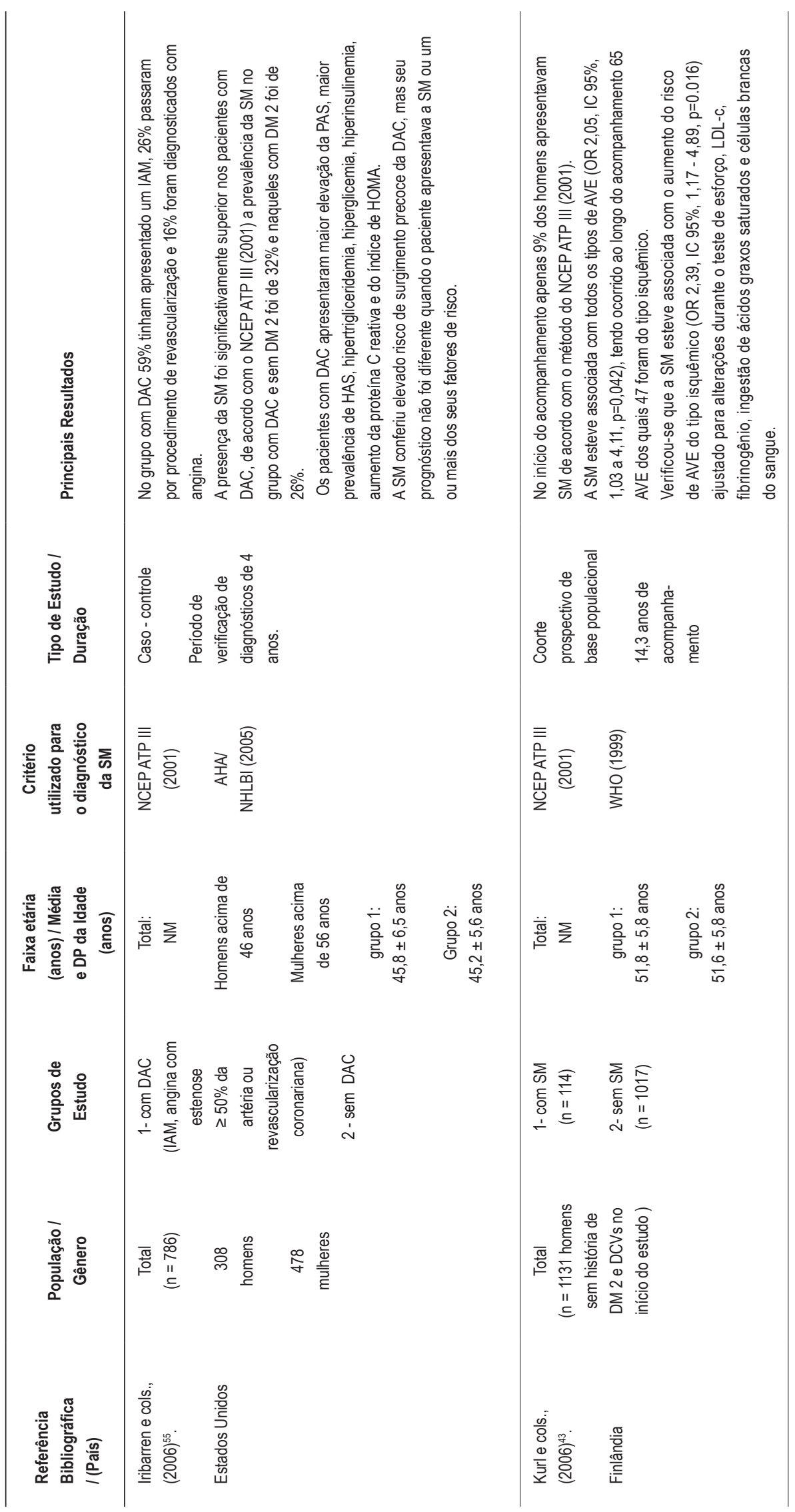




\section{Atualização Clínica}

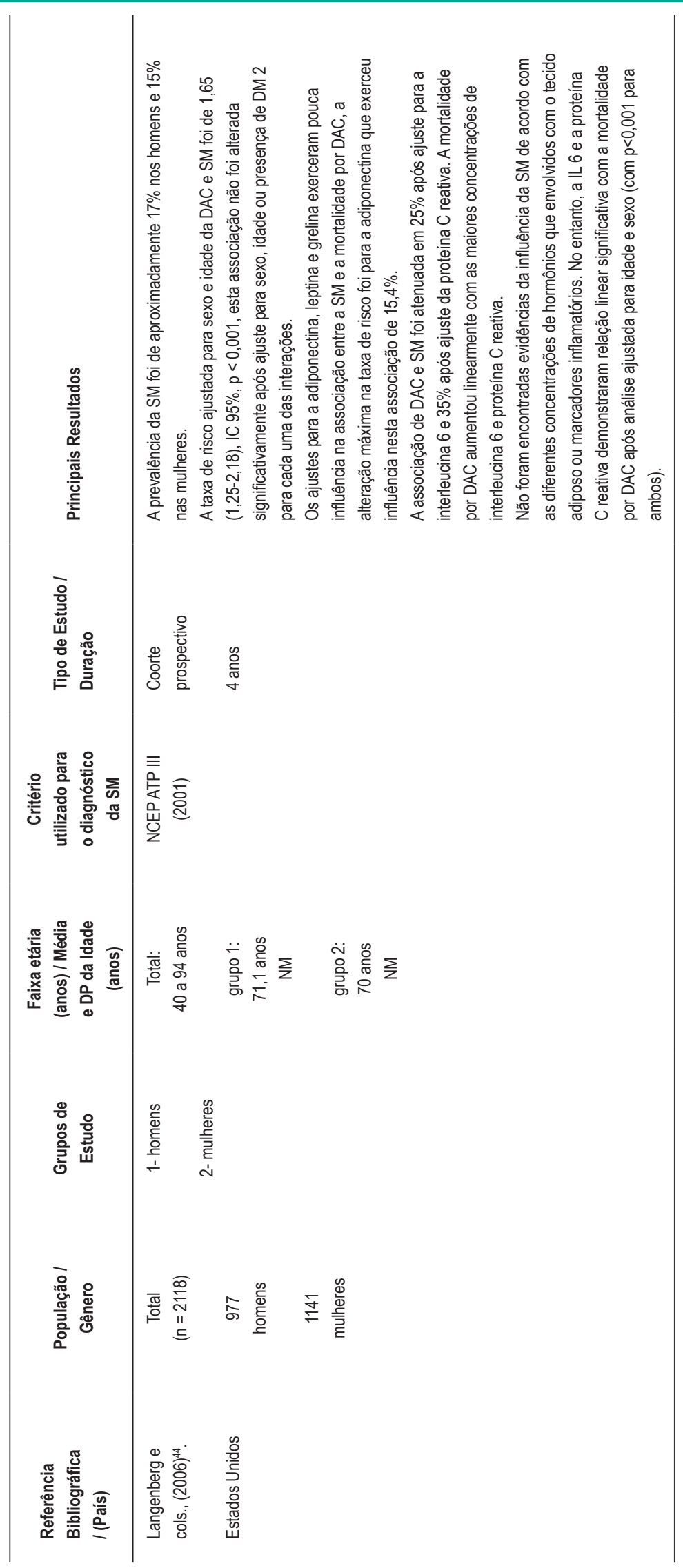




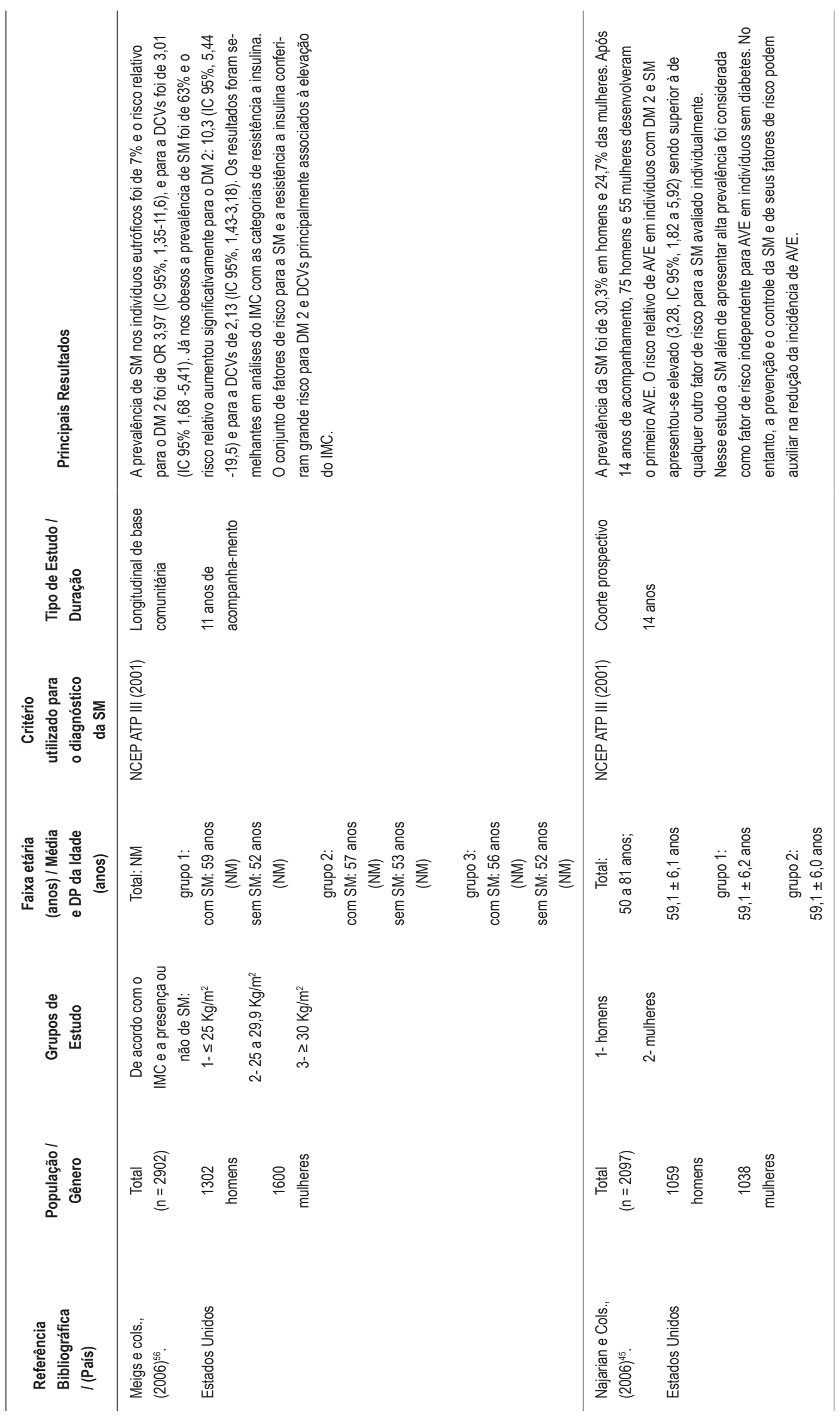




\section{Atualização Clínica}

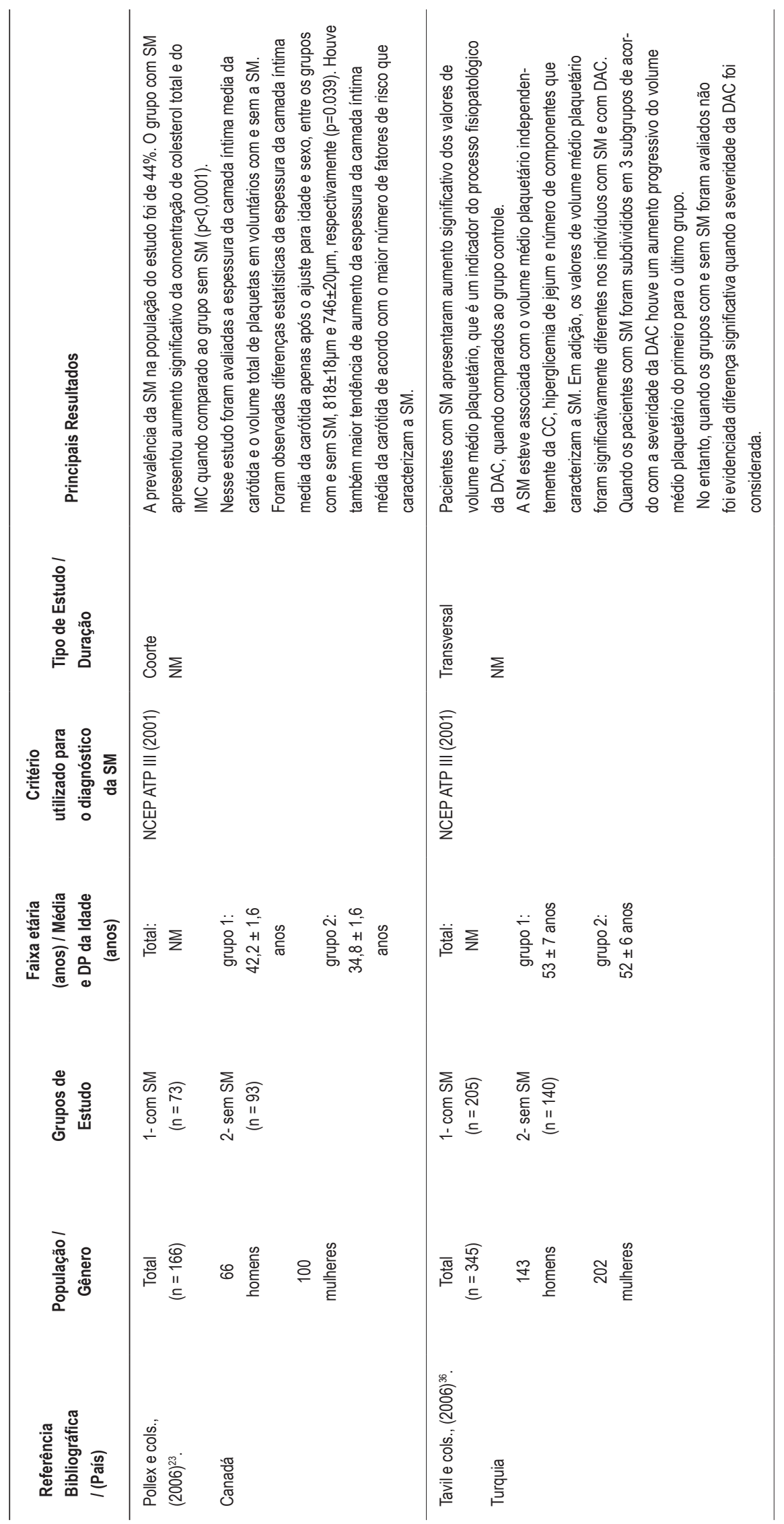




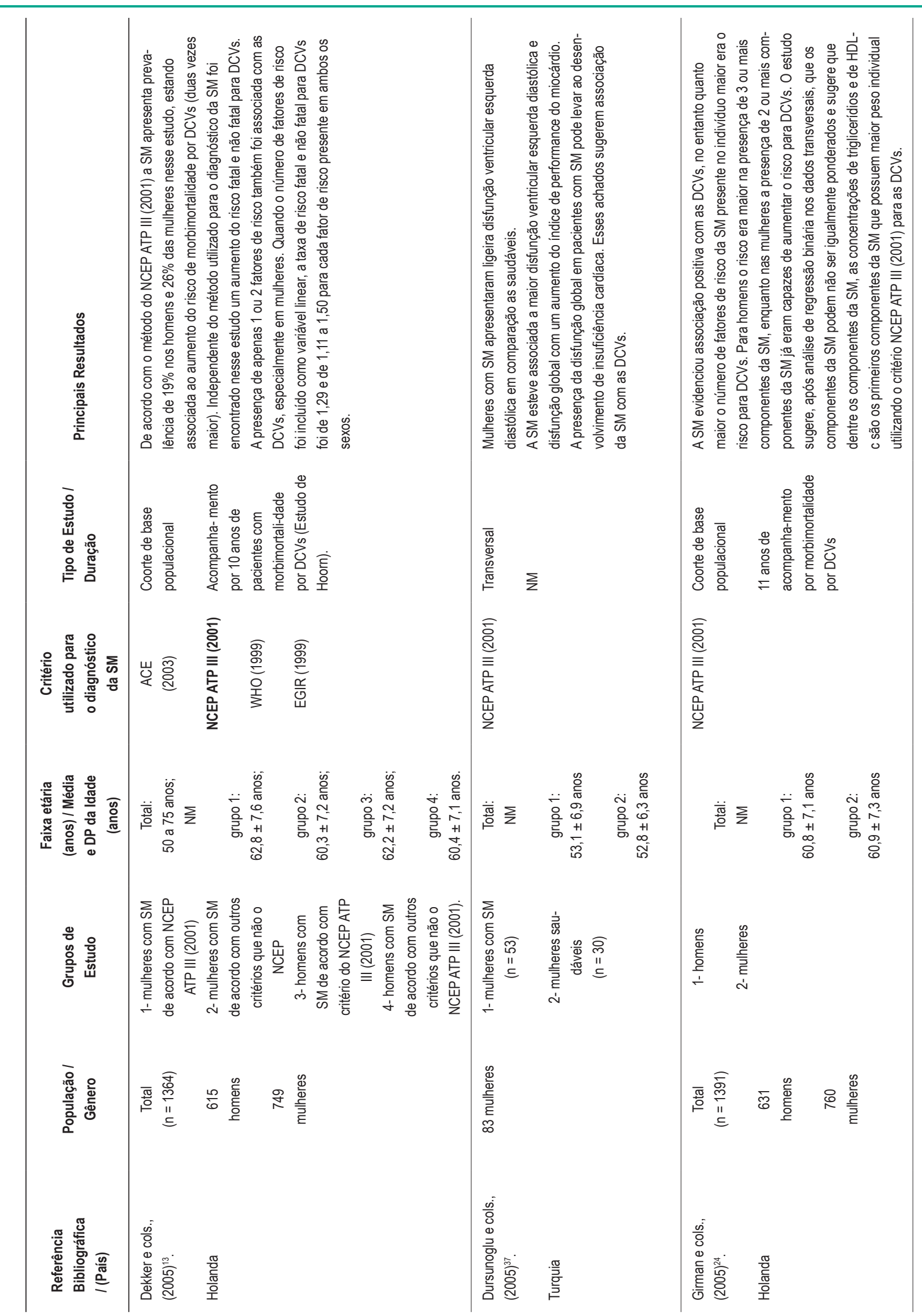




\section{Atualização Clínica}

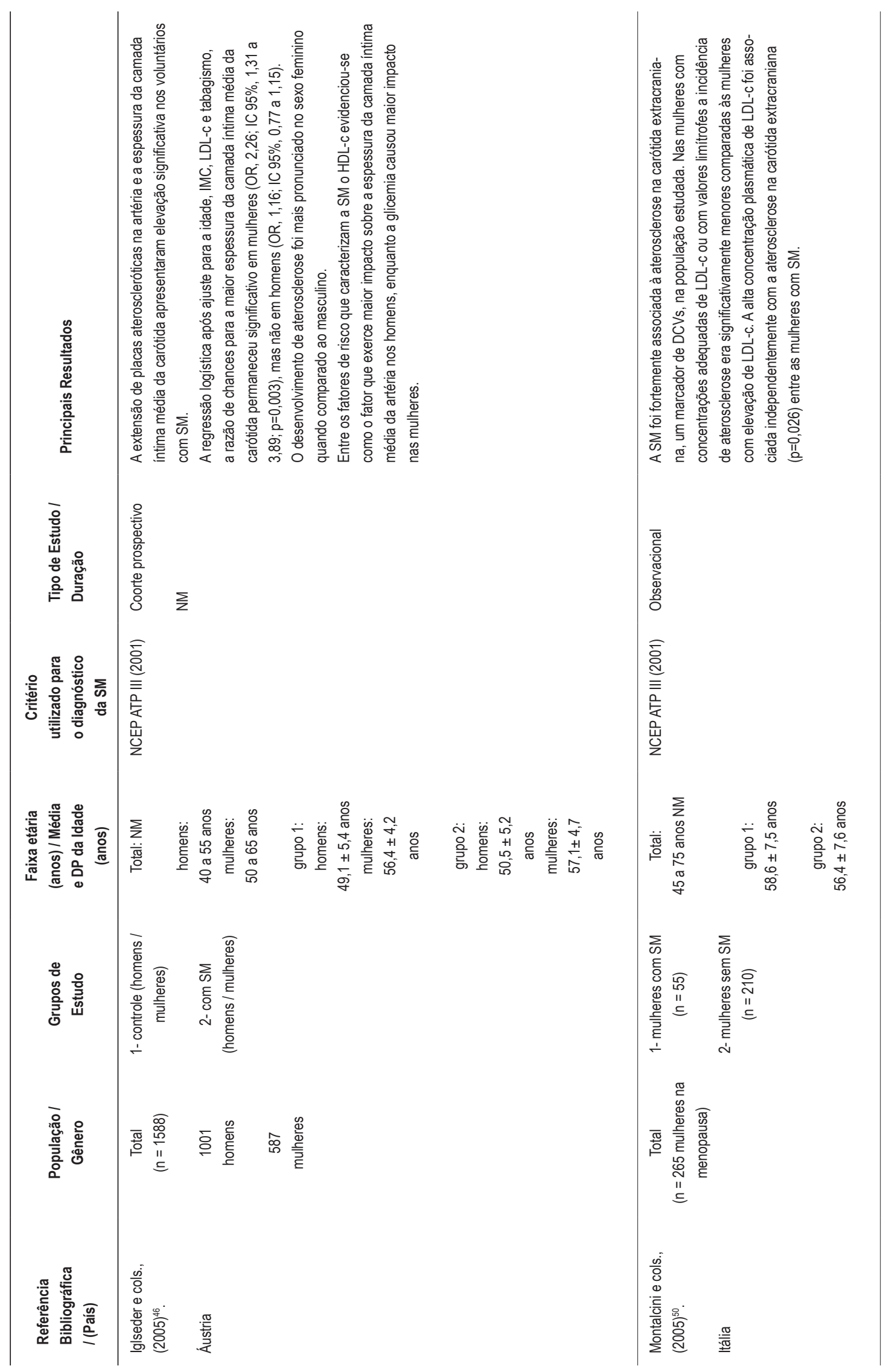




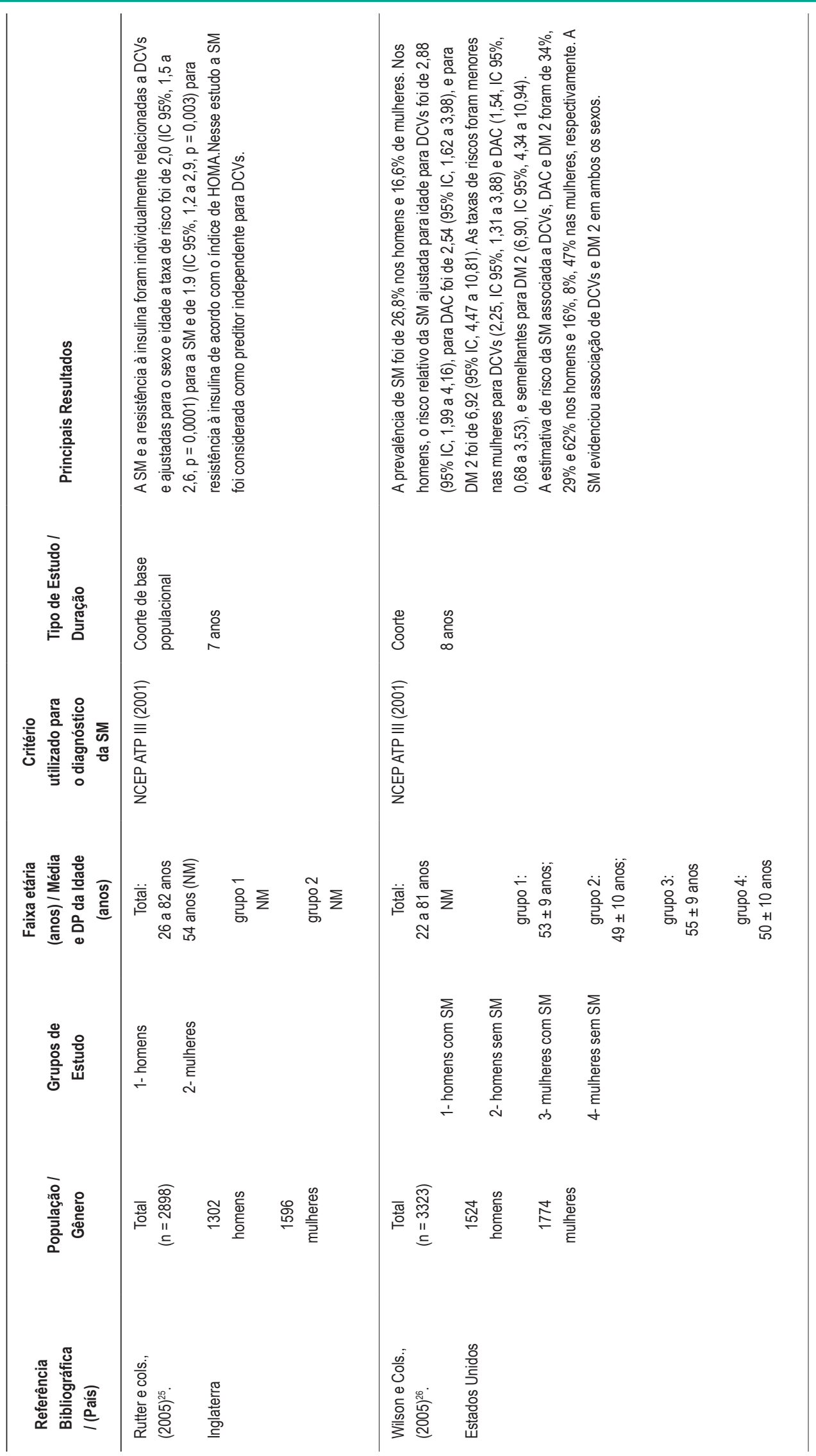




\section{Atualização Clínica}

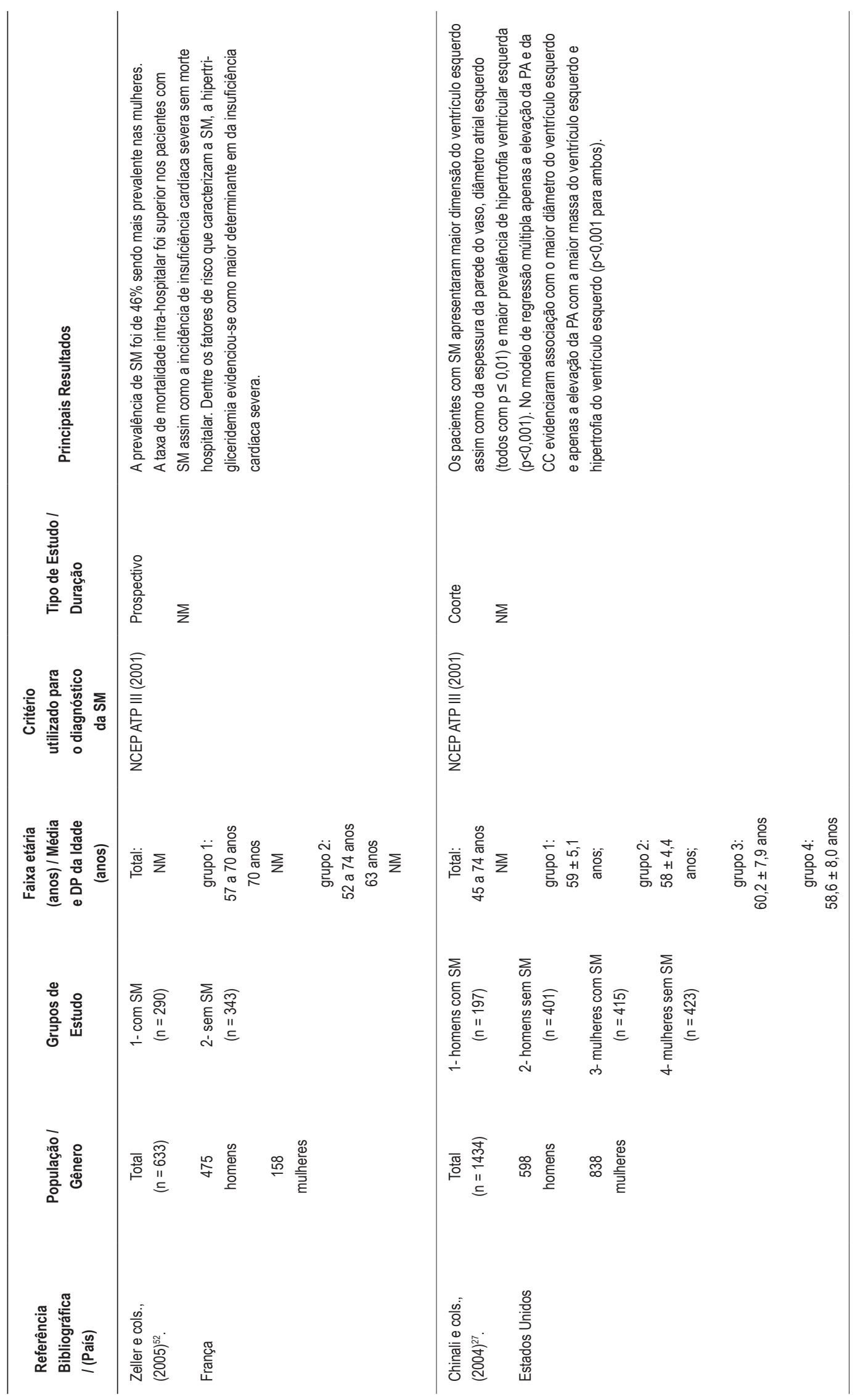




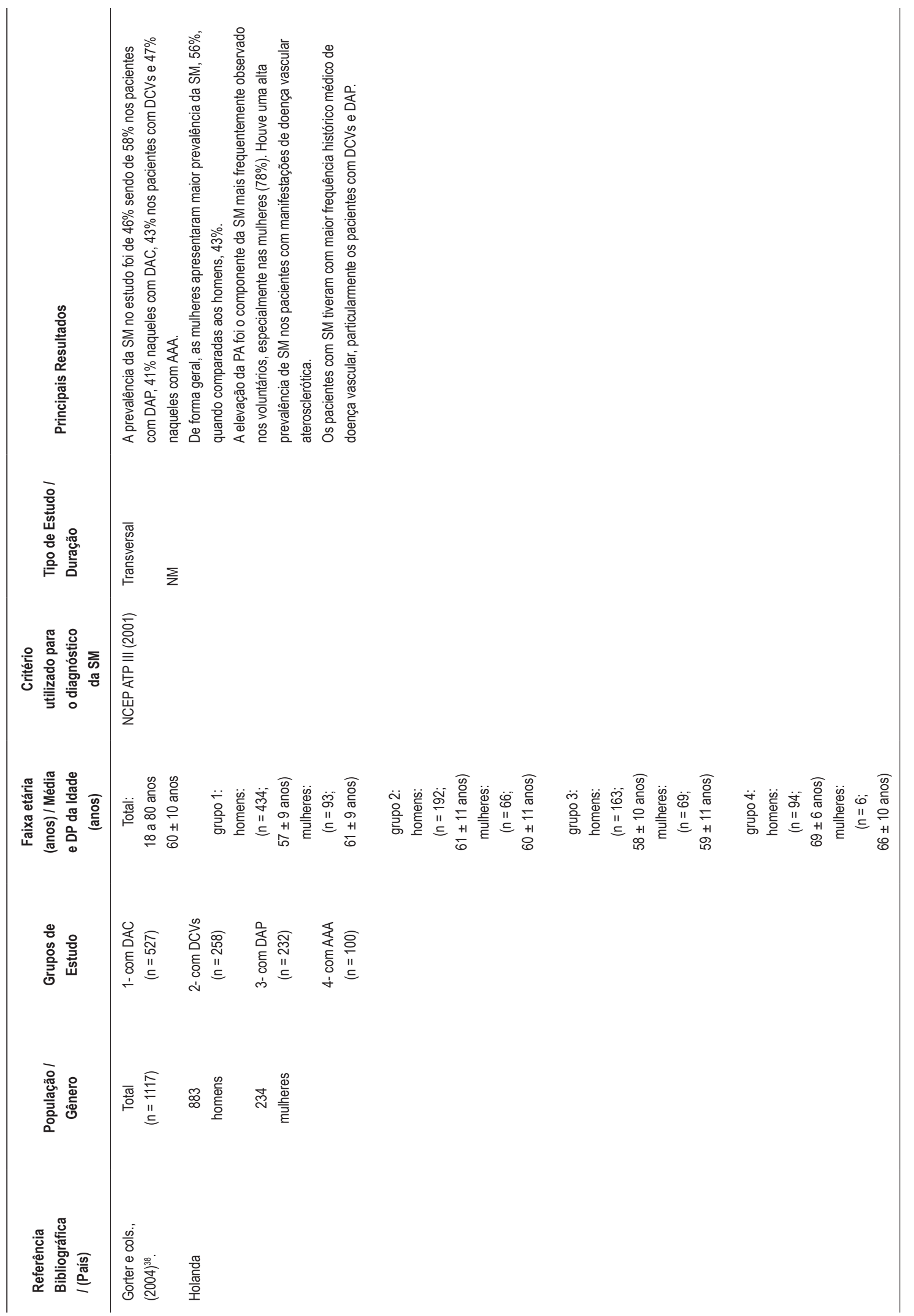




\section{Atualização Clínica}

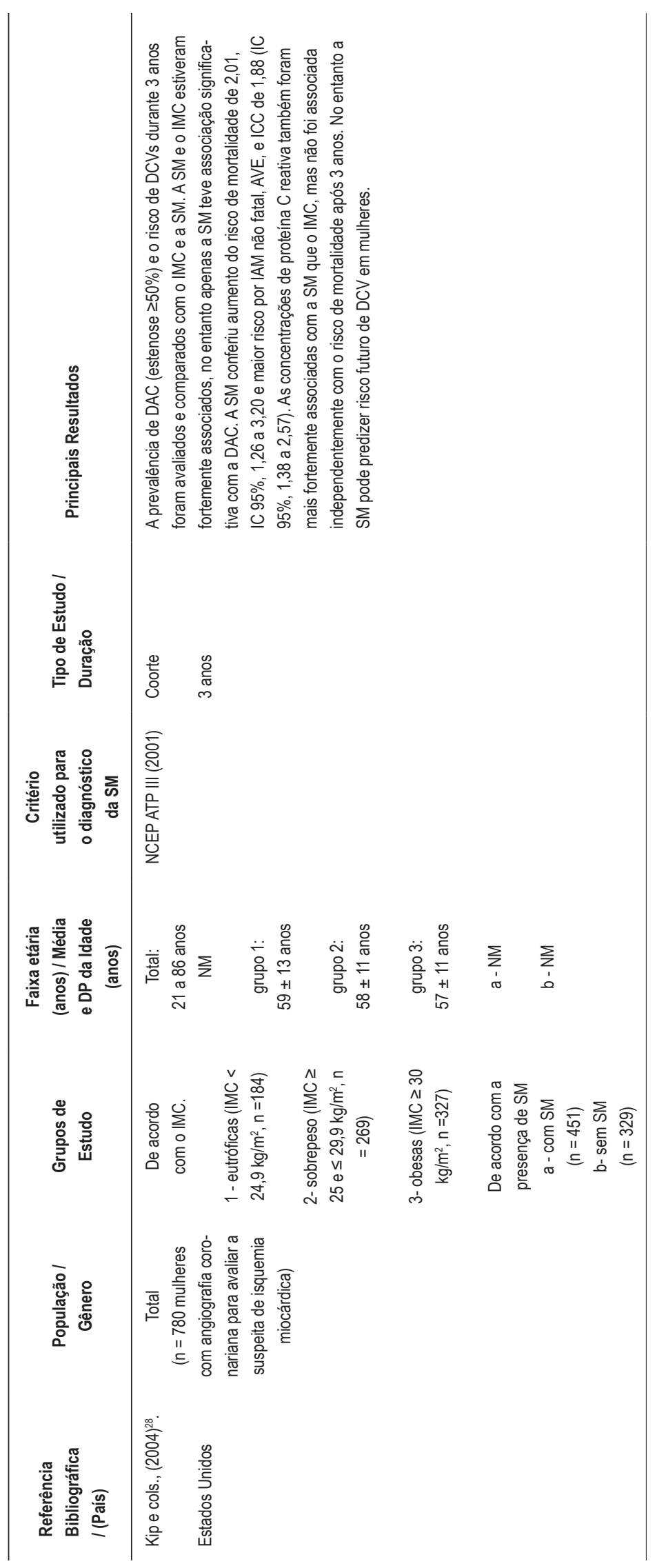




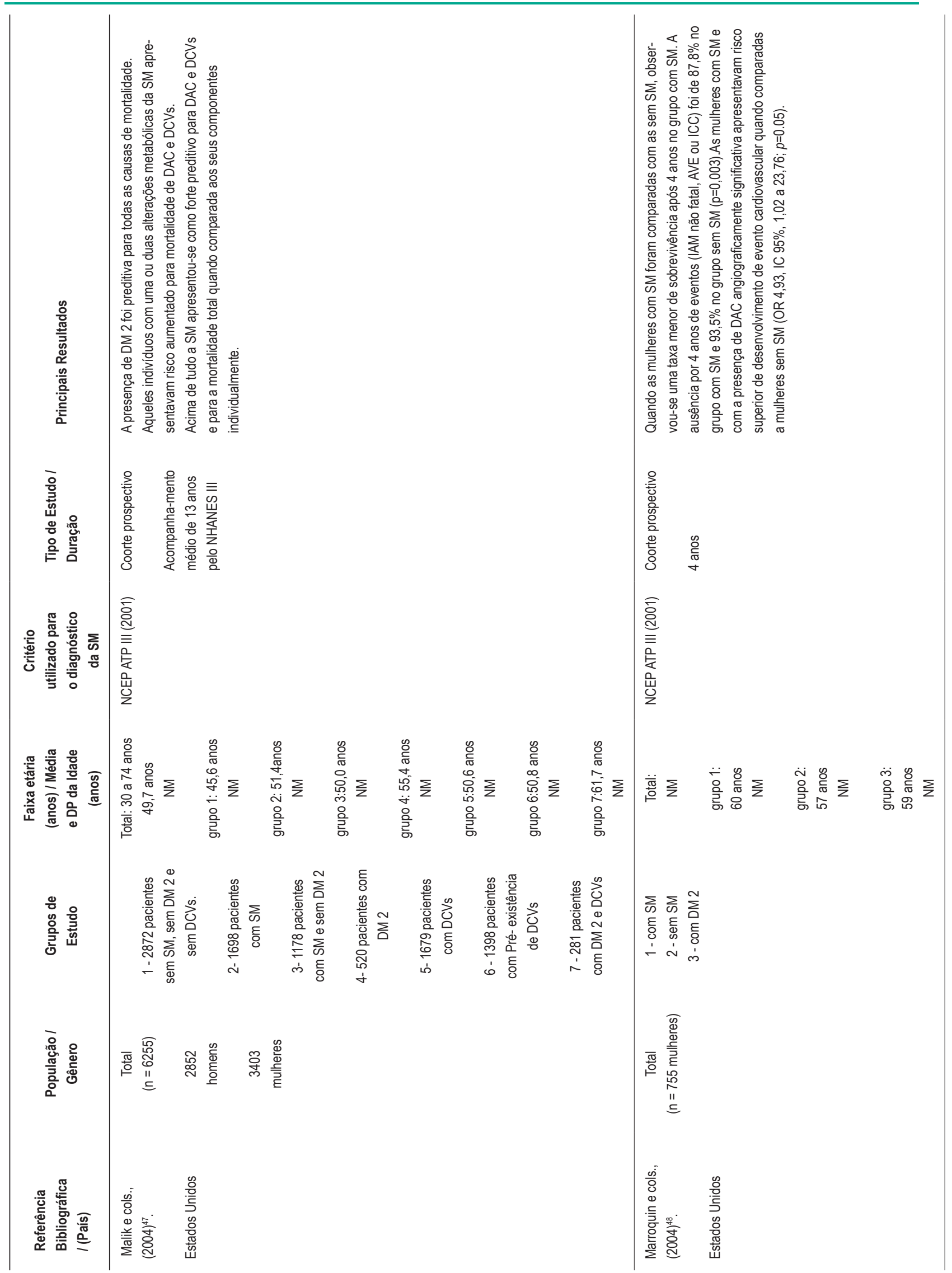




\section{Atualização Clínica}

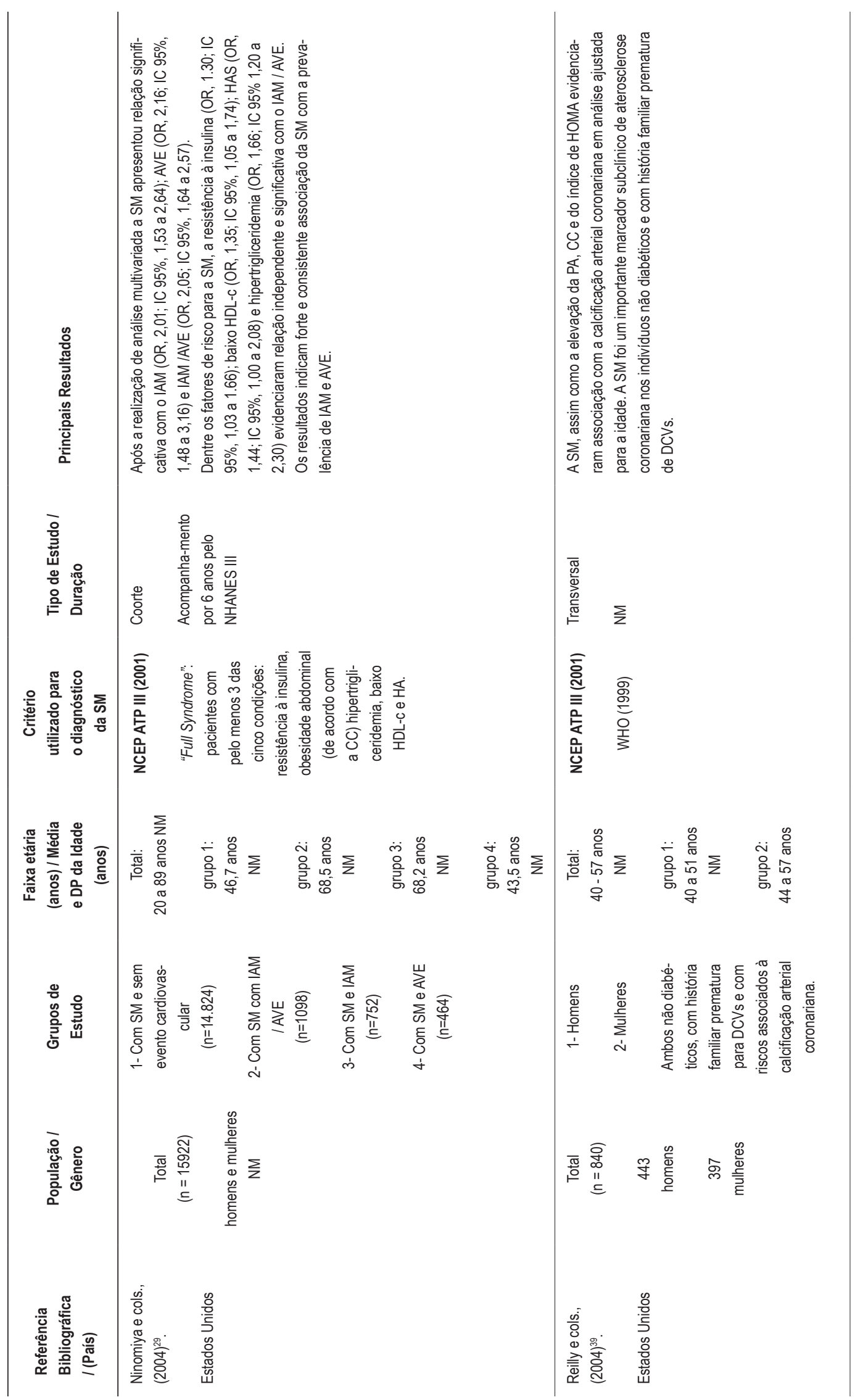




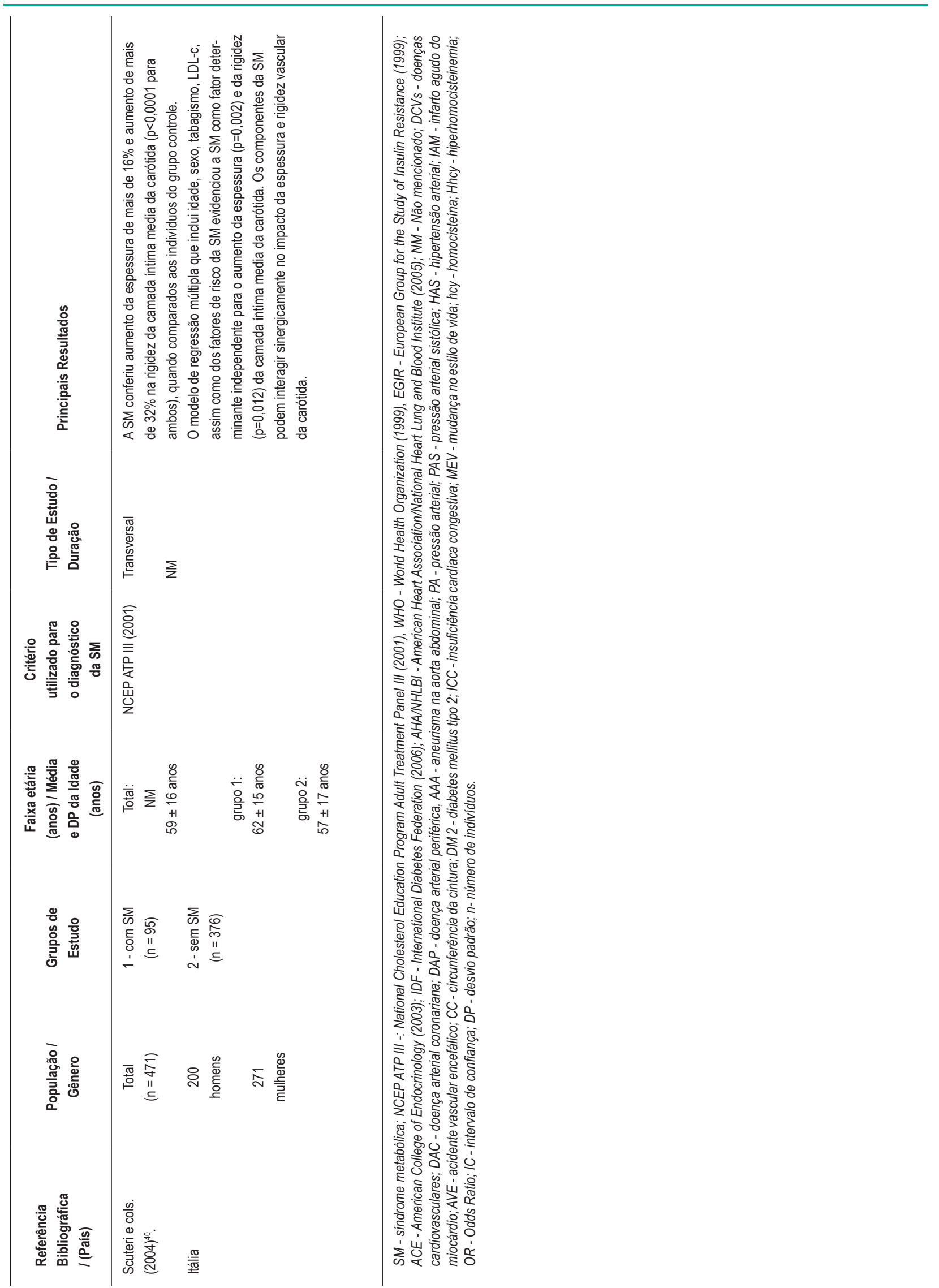




\section{Atualização Clínica}

\begin{tabular}{|c|c|c|c|c|c|c|c|c|c|}
\hline \multirow[b]{2}{*}{ Study or Subgroup } & \multicolumn{2}{|c|}{ Positivo } & \multicolumn{2}{|c|}{ Negativo } & \multirow[b]{2}{*}{ Weight } & \multirow{2}{*}{$\begin{array}{c}\text { Risk Ratio } \\
\text { M-H, Fixed, } 95 \% \mathrm{CI}\end{array}$} & \multirow{2}{*}{\multicolumn{3}{|c|}{$\begin{array}{c}\text { Risk Ratio } \\
\text { M-H, Fixed, } 95 \% \mathrm{Cl}\end{array}$}} \\
\hline & Events & Total & Events & Total & & & & & \\
\hline Athyros 2007 & 1279 & 2369 & 1041 & 7300 & $16.2 \%$ & $3.79[3.54,4.05]$ & & - & \\
\hline Brevetti 2006 & 27 & 79 & 15 & 75 & $0.5 \%$ & $1.71[0.99,2.95]$ & & - & \\
\hline Carminit 2008 & 98 & 137 & 95 & 149 & $2.9 \%$ & $1.12[0.96,1.32]$ & & 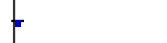 & \\
\hline Eberly 2006 & 1532 & 4588 & 1431 & 6362 & $38.2 \%$ & $1.48[1.40,1.58]$ & & $\square$ & \\
\hline Empana2007 & 84 & 674 & 383 & 4911 & $2.9 \%$ & $1.60[1.28,2.00]$ & & - & \\
\hline Hajer 2007 & 195 & 886 & 172 & 1174 & $4.7 \%$ & $1.50[1.25,1.81]$ & & \% & \\
\hline Iribarren 2006 & 209 & 267 & 184 & 519 & $4.0 \%$ & $2.21[1.93,2.52]$ & & r & \\
\hline Kasai 2008 & 98 & 167 & 232 & 489 & $3.8 \%$ & $1.24[1.06,1.45]$ & & ${ }^{*}$ & \\
\hline Kip 2004 & 209 & 259 & 81 & 329 & $2.3 \%$ & $3.28[2.69,4.00]$ & & r & \\
\hline Langenberg 2006 & 65 & 337 & 228 & 1781 & $2.3 \%$ & $1.51[1.17,1.93]$ & & - & \\
\hline Malik 2004 & 336 & 1698 & 132 & 4557 & $2.3 \%$ & $6.83[5.63,8.29]$ & & - & \\
\hline Marroquin 2004 & 142 & 430 & 85 & 325 & $3.1 \%$ & $1.26[1.01,1.58]$ & & 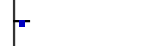 & \\
\hline Meigs 2006 & 123 & 804 & 135 & 2098 & $2.4 \%$ & $2.38[1.89,2.99]$ & & - & \\
\hline Montalcini 2005 & 44 & 55 & 84 & 210 & $1.1 \%$ & $2.00[1.62,2.47]$ & & $=$ & \\
\hline Najarian 2006 & 156 & 577 & 76 & 1520 & $1.3 \%$ & $5.41[4.18,6.99]$ & & - & \\
\hline Noto 2008 & 27 & 157 & 44 & 528 & $0.6 \%$ & $2.06[1.32,3.22]$ & & - & \\
\hline Reilly 2004 & 60 & 208 & 19 & 632 & $0.3 \%$ & $9.60[5.87,15.69]$ & & - & \\
\hline Scuteri 2004 & 38 & 95 & 113 & 376 & $1.5 \%$ & $1.33[1.00,1.78]$ & & + & \\
\hline Skoumas 2007 & 45 & 295 & 46 & 411 & $1.2 \%$ & $1.36[0.93,2.00]$ & & - & \\
\hline Wierzbicki 2008 & 93 & 176 & 105 & 224 & $2.9 \%$ & $1.13[0.93,1.37]$ & & + & \\
\hline Wilson I 2005 & 103 & 613 & 91 & 2710 & $1.1 \%$ & $5.00[3.83,6.54]$ & & - & \\
\hline Zeller 2005 & 114 & 290 & 152 & 343 & $4.4 \%$ & $0.89[0.74,1.07]$ & & 1 & \\
\hline Total $(95 \% \mathrm{Cl})$ & & 15161 & & 37023 & $100.0 \%$ & $2.13[2.06,2.20]$ & & 1 & \\
\hline Total events & 5077 & & 4944 & & & & & & \\
\hline $\begin{array}{l}\text { Heterogeneity: } \mathrm{Chi}^{2}= \\
\text { Test for overall effect: }\end{array}$ & $\begin{array}{l}93.73, \mathrm{df} \\
Z=45.45\end{array}$ & $\begin{array}{l}=21(P \\
(P<0.0\end{array}$ & $\begin{array}{l}<0.00001 \\
0001)\end{array}$ & 1); $1^{2}=9$ & & & $\begin{array}{cc}0.001 & 0.1 \\
\text { avours experimental }\end{array}$ & $\begin{array}{l}10 \\
\text { Favours cont }\end{array}$ & $\begin{array}{l}1000 \\
\text { trol }\end{array}$ \\
\hline
\end{tabular}

Fig. 1 - Heterogeneidade e risco para o desenvolvimento da doença vascular oclusiva ou coronariana ou acidente vascular encefálico entre grupos com e sem SM.

mas também por ele ser um critério largamente utilizado em publicações científicas.

Individualmente, os componentes da SM são fatores de risco independentes para o desenvolvimento de doença cardiovascular aterosclerótica ${ }^{10,13,20,23,24,40,42}$. Os diferentes critérios existentes para o diagnóstico da SM são baseados no princípio de que seus fatores de risco possam interagir sinergicamente, ou ainda de maneira a ampliar o risco para DAC e doença aterosclerótica. Alguns estudos demonstraram que quanto maior o número dos componentes da SM em um indivíduo, maior o risco ou a extensão da patologia associada a $\mathrm{SM}^{15,23,24,31}$.

Os estudos selecionados nesta revisão sistemática evidenciaram a associação da SM com o desenvolvimento ou a mortalidade por DCV $10,13,20,24,25,29,41,47,49,51$, DAC $22,28,31,44,47,55$, diabetes mellitus 2 (DM 2) 20,26,56 e acidente vascular encefálico $(\mathrm{AVE})^{29,43,45}$. Alguns estudos ainda apontam que os indivíduos com SM apresentam maior mortalidade por DCVs ${ }^{20,41}$ e maior prevalência de $\mathrm{AVE}^{43}$ independentemente da presença de intolerância à glicose ou DM 20,41,45 .

Dentre os artigos selecionados, 4 avaliaram a espessura médio-íntima da carótida $23,40,42,46$, indicando associação do processo aterotrombótico com a SM, sendo a elevação da pressão arterial $^{42}$, a diminuição da concentração de HDL-c em homens e a hiperglicemia de jejum em mulheres ${ }^{46}$ os componentes da SM mais importantes nessa associação. Foi descrito que quanto maior o número de fatores de risco que caracterizam a SM maior era a tendência de aumento da espessura médio-íntima da carótida ${ }^{23}$.

Alguns estudos relatam a associação da SM com a elevação do índice de massa corpórea (IMC) $)^{28,30,56}$; ainda foi destacado que os indivíduos com SM e maior IMC apresentaram maior risco de DM 2 e $\mathrm{DCVs}^{56}$. Outros estudos evidenciaram a associação da SM com o aumento da atividade de células de adesão molecular, hipoadiponectinemia ${ }^{32}$, e o aumento das concentrações de LDL-oxidada ${ }^{34,53}$ e proteína $C$ reativa ${ }^{35,55}$. Um estudo relata que houve aumento da mortalidade por DAC em pacientes com SM e elevação da concentração de proteína $\mathrm{C}$ reativa ${ }^{44}$.

Estudos que avaliaram a concentração circulante de LDLoxidada demonstraram que dentre os fatores de risco que caracterizam a SM, aqueles que apresentam maior associação com a oxidação dessa lipoproteína são a hiperglicemia de jejum, hipertrigliceridemia, baixa concentração de HDL-c e a obesidade abdominal ${ }^{34,53}$. O Odds Ratio adaptado para a 
incidência dicotômica dos fatores de risco que caracterizam a SM, versus o quinto quintil de LDL-oxidada foram de 2,1 (IC 95\%, 1,2 - 3,6) para a obesidade abdominal; 2,4 (IC 95\%, 1,5 - 3,8) para hiperglicemia de jejum e 2,1 (IC 95\%, 1,1 - 4,0) para a hipertriglicerídemia ${ }^{53}$.

Apesar da hiper-homocisteinemia (Hhcy) ser um conhecido marcador de doenças vasculares oclusivas, ainda existem poucos estudos que avaliam sua associação com a SM. Dentre os estudos selecionados nesta revisão sistemática, apenas dois avaliaram a associação da Hhcy com a SM e seus fatores de risco ${ }^{19,49}$. Um estudo recente demonstrou que a concentração plasmática de homocisteína (hcy) foi superior nos indivíduos com SM quando comparados aos sem SM. Sua concentração ainda aumentava de acordo com o número de fatores de risco que caracterizam a SM. Quando os indivíduos sem a SM foram comparados com aqueles que apresentavam os cinco fatores de risco para a SM, a concentração plasmática de hcy aumentou significativamente (de 12,7 $\mu \mathrm{mol} / \mathrm{l}$ para $15,9 \mu \mathrm{mol} / \mathrm{I}^{19}$. Tais achados sugerem que a Hhcy e a SM apresentam efeito sinérgico no aumento do risco para as doenças vasculares oclusivas.

Dos estudos selecionados nesta revisão, um não evidenciou a associação da SM com a DAC ${ }^{54}$, e outro não evidenciou associação da SM com a formação de placa ateromatosa ${ }^{18}$. No entanto, a maioria dos estudos demonstraram a associação da SM ou de alguns de seus componentes com o maior risco de morbimortalidade geral e por DAC. Por isso, devemos evidenciar a importância de atuar na prevenção e controle da SM, assim como dos fatores de risco que a ela estão associados, para que seja possível trabalhar na redução de DCVs, principal causa de mortalidade mundial.

A heterogeneidade entre os estudos selecionados pode ser observada na figura $1 \mathrm{e}$, provavelmente, é decorrente dos diferentes marcadores utilizados para o diagnóstico da doença vascular oclusiva ou coronariana. Também foi demonstrada uma maior probabilidade (risco $=2,13$ ) dos os indivíduos com SM desenvolverem a doença vascular ou coronariana. Mas, os resultados continuam contraditórios, sendo necessária a realização de mais estudos para a obtenção de resultados mais consistentes.

A adoção, preferencialmente desde a infância e por toda a população, de estilos de vida saudáveis, como uma dieta equilibrada e a prática regular de atividade física, é um componente básico da prevenção da SM. Os benefícios da prática regular de exercícios físicos sob a redução da morbimortalidade por DAC têm sido evidenciados na literatura científica como parte da mudança do estilo de vida (MEV), juntamente com a redução de deposição de gordura corporal, principalmente aquela localizada na região abdominal, que representa fator de risco importante para o diagnóstico da SM. O sucesso da intervenção no controle e tratamento da SM está intimamente relacionado com a MEV, sendo a terapia não-medicamentosa de primeira escolha para o seu tratamento, com a realização de um plano alimentar para a redução de peso corporal associado ao exercício físico ${ }^{57}$, além do combate ao tabagismo, ao uso abusivo de álcool e ao estresse. Essas modificações comportamentais já poderão melhorar a qualidade e expectativa de vida dos indivíduos com SM ${ }^{11}$.
Apesar da MEV ser fundamental na prevenção e tratamento da SM, apenas um estudo evidenciou em sua conclusão a importância da MEV para a redução da morbimortalidade por DAC e doenças vasculares oclusivas nos pacientes com SM ${ }^{10}$. Outro estudo evidenciou apenas a importância da prevenção da SM e dos fatores de risco que a caracterizam para a redução da incidência de $\mathrm{AVE}^{29}$.

Portanto, a fim de alcançarmos a remissão da SM e minimizarmos a prevalência das DAC e doenças vasculares oclusivas, os profissionais da saúde devem incentivar práticas alimentares saudáveis, como a diminuição do consumo de gorduras saturadas e hidrogenadas, o aumento do consumo de frutas, verduras, fibras e cereais integrais e de mudanças no estilo de vida, como a cessação do tabagismo, que constitui medida fundamental e prioritária na prevenção dos componentes isolados da SM. Além disso, a prática de exercícios físicos aeróbios promove a redução das concentrações plasmáticas de triglicerídios e o aumento das concentrações de HDL-C ${ }^{58}$, fatores de risco importantes que caracterizam a SM.

Uma dieta equilibrada associada à prática regular de atividade física seria capaz de promover a redução do peso corporal, além de melhorar o quadro clínico dos pacientes com SM, proporcionando a diminuição dos riscos e da morbimortalidade por DAC e doenças vasculares oclusivas.

\section{Conclusão}

A compreensão sobre a agregação dos componentes da SM, bem como os mecanismos fisiopatológicos que as desencadeiam, ainda não foi alcançada. Mas, apesar dos relatos na literatura científica serem controversos e da heterogeneidade entre os estudos, observou-se nesta revisão o grande impacto da SM na ocorrência de DAC e doenças vasculares oclusivas.

Portanto, é de suma importância a realização de estudos randomizados, que utilizem marcadores mais fidedignos para o diagnóstico da DAC e das doenças vasculares oclusivas, gerando resultados mais consistentes.

\section{Agradecimentos}

Agradecemos ao apoio do Conselho Nacional de Desenvolvimento Científico e Tecnológico (CNPq) e da Fundação Carlos Chagas Filho de Amparo à Pesquisa do Rio de Janeiro (FAPERJ).

\section{Potencial Conflito de Interesses}

Declaro não haver conflito de interesses pertinentes.

\section{Fontes de Financiamento}

O presente estudo foi financiado pelo CNPq e FAPERJ.

\section{Vinculação Acadêmica}

Não há vinculação deste estudo a programas de pósgraduação. 


\section{Atualização Clínica}

\section{Referências}

1. Executive Summary of the Third Report of the National Cholesterol Education Program (NCEP) Expert Panel on Detection, Evaluation, and Treatment of High Blood Cholesterol in Adults (Adult Treatment Panel III). JAMA. 2001; 285: 2486-97.

2. World Health Organization. Definition, diagnosis and classification of diabetes mellitus and its complications: report of WHO a Consultation. Part 1: diagnosis and classification of diabetes mellitus. Genebra; 1999.

3. Balkau B, Charles MA. Comment on the provisional report from the WHO consultation. European Group for the Study of Insulin Resistance (EGIR). Diabet Med. 1999; 16 (5): 442-3.

4. Grundy SM, Cleeman JI, Daniels SR, Donato KA, Eckel RH, Franklin BA, et al. Diagnosis and management of the metabolic syndrome: an American Heart Association/National Heart, Lung, and Blood Institute Scientific Statement. Circulation. 2005; 112 (17): 2735-52.

5. International Diabetes Federation (IDF). The IDF consensus worldwide definition of the metabolic syndrome. 2006 [Acessed on 2007 Oct 10]. Available from: http://www.idf.org/webdata/docs/Meta_syndrome_ definition.pdf.

6. Eckel RH, Grundy SM, Zimmet PZ: The metabolic syndrome. Lancet. 2005; 365: 1415-28.

7. Grundy SM. Metabolic syndrome: what is it and how should I treat it? ACC Curr J Rev. 2003; 12 (3): 37-40.

8. Resnick HE, Jones K, Ruotolo G, Jain AK, Henderson J, Lu W, et al. Insulin resistance, the metabolic syndrome, and risk of incident cardiovascular disease in nondiabetic american indians. Diabetes Care. 2003; 26: 861-7.

9. Pereira MA, Jacobs DRJ, Van Horn L, Slattery ML, Kartashov AI, Ludwig DS. Dairy consumption, obesity, and the insulin resistance syndrome in young adults: the Cardia Study. JAMA. 2002; 287 (16): 2081-9.

10. Athyros VG, Ganotakis ES, Elisaf MS, Liberopoulos EN, Goudevenos IA, Karagiannis A. Prevalence of vascular disease in metabolic syndrome using three proposed definitions. Int J Cardiol. 2007; 117: 204-10.

11. Lerário DDG, Gimeno SG, Franco LJ, lunes M, Ferreira SRG. Excesso de peso e gordura abdominal para a síndrome metabólica em nipo-brasileiros. Rev Saude Publica. 2002; 36 (1): 4-11.

12. Pousada JMDC, Britto MMS, Cruz T, Lima ML, Lessa I, Lemaire DC, et al. The metabolic syndrome in spanish migrants to Brazil: unexpected results. Diabetes Res Clin Pract. 2006; 72: 75-80.

13. Dekker JM, Girman C, Rhodes T, Nijpels G, Stehouwer CDA, Bouter LM, et al. Metabolic syndrome and 10-year cardiovascular disease risk in the Hoorn study. Circulation. 2005; 112: 666-73.

14. American College of Endocrinology. Executive summary of the position statement on the insulin resistance syndrome. Endocr Pract. 2003; 9 (3):237-9.

15. Hamburg NM, Larson MG, Vita JA, Vasan RS, Keyes MJ, Widlansky ME, et al. Metabolic syndrome, insulin resistance, and brachial artery vasodilator function in framingham offspring participants without clinical evidence of cardiovascular disease. Am J Cardiol. 2008; 101 :82-8.

16. Review Manager (RevMan) [Computer program]. Version 5.0. Copenhagen: The Nordic Cochrane Centre, The Cochrane Collaboration, 2008.

17. Higgins JP, Thompson SG, Deeks JJ, Altman DG. Measuring inconsistency in meta-analyses. BMJ. 2003;327: 557-60.

18. Wierzbicki AS, Nishtar S, Lumb PJ, Lambert-Hammill M, Crook MA, Marber MS, et al. Waist circumference, metabolic syndrome and coronary artery disease in a Pakistani cohort. Int J Cardiol. 2008; 128: 77-82.

19. Hajer GR, Graaf YVD, Olijhoek JK, Verhaar MC, Visseren FLJ. Levels of homocysteine are increased in metabolic syndrome patients but are not associated with an increased cardiovascular risk, in contrast to patients without the metabolic syndrome. Heart. 2007; 93: 216-20.

20. Lorenzo C, Williams K, Hunt KJ, Haffner SM. The National Cholesterol Education Program-Adult Treatment Panel III, International Diabetes Federation, and World Health Organization Definitions of the Metabolic
Syndrome as Predictors of Incident Cardiovascular Disease and Diabetes. Diabetes Care. 2007; 30: 8-13.

21. Stein E, Kushnerc H, Giddingd S, Falknera B. Plasma lipid concentrations in nondiabetic African American adults: associations with insulin resistance and the metabolic syndrome. Metabolism. 2007; 56: 954-60.

22. Eberly LE, Prineas R, Cohen JD, Vazquez G, Zhi X, Neaton JD, et al. Metabolic syndrome. Diabetes Care. 2006; 29: 123-30.

23. Pollex RL, Al-Shali KZ, House AA, Spence JD, Fenster A, Mamakeesick M, et al. Relationship of the metabolic syndrome to carotid ultrasound traits. Cardiovasc Ultrasound. 2006; 4: 28.

24. Girman CJ, Dekker JM, Rhodes T, Nijpels G, Stehouwer CDA, Bouter LM, et al. An exploratory analysis of criteria for the metabolic syndrome and its prediction of long-term cardiovascular outcomes: the Hoorn Study. Am J Epidemiol. 2005; 162: 438-47.

25. Rutter MK, Meigs JB, Sullivan LM, D'Agostino RB, Wilson PW. Insulin resistance, the metabolic syndrome, and incident cardiovascular events in the Framingham Offspring Study. Diabetes. 2005; 54: 3252-7.

26. Wilson MPWF, D'Agostino RB, Parise H, Sullivan L, James B. Metabolic syndrome as a precursor of cardiovascular disease and type 2 Diabetes Mellitus. Circulation. 2005; 112: 3066-72.

27. Chinali M, Devereux RB, Howard BV, Roman MJ, Bella JN, Liu JE, et al. Comparison of cardiac structure and function in american indians with and without the metabolic syndrome: the Strong Heart Study. Am J Cardiol. 2004; 93: $40-4$.

28. Kip KE, Marroquin OC, Kelley DE, Johnson BD, Kelsey SF, Shaw LJ, et al. Clinical importance of obesity versus the metabolic syndrome in cardiovascular risk in women: a report from the Women's Ischemia Syndrome Evaluation (WISE) Study. Circulation. 2004; 109: 706-13.

29. Ninomiya J, L'Italien G, Criqui MH, Whyte JL, Gamst A, Chen R. Association of the Metabolic syndrome with history of myocardial infarction and stroke in the Third National Health and Nutrition Examination Survey. Circulation. 2004; 109: 42-6.

30. Caminiti G, Volterrani M, Marazzi G, Massaro R, Vitale C, Gatta L, et al. Metabolic syndrome predicts lower functional recovery in female but not in male patients after an acute cardiac event. Int J Cardiol. 2008. (In press).

31. Kasai T, Miyauchi K, Kubota N, Tamura H, Kojima T, Yokoyama K, et al. The relationship between the metabolic syndrome defined by various criteria and the extent of coronary artery disease. Atherosclerosis. 2008; 197: 944-50.

32. Gomez Rosso L, Benitez MB, Fornari MC, Berardi V, Lynch S, Schreier L, et al. Alterations in cell adhesion molecules and other biomarkers of cardiovascular disease in patients with metabolic syndrome. Atherosclerosis. 2008; 199: 415-23.

33. Ryan MC, Farin HMF, Abbasi F, Reaven GM. Comparison of waist circumference versus body mass index in diagnosing metabolic syndrome and identifying apparently healthy subjects at increased risk of cardiovascular disease. Am J Cardiol. 2008; 102: 40-6.

34. Lapointe A, Couillard C, Piche ME, Weisnagel SJ, Bergeron J, Nadeau A, et al. Circulating oxidized LDL is associated with parameters of the metabolic syndrome in postmenopausal women. Atherosclerosis. 2007; 191 (2): 362-8.

35. Brevetti G, Schiano V, Sirico G, Giugliano G, Laurenzano E, Chiariello M. Metabolic syndrome in peripheral arterial disease: relationship with severity of peripheral circulatory insufficiency, inflammatory status, and cardiovascular comorbidity. J Vasc Surg. 2006; 44: 101-7.

36. Tavil Y, Sen N, Yazici HU, Hizal F, Abaci A, Cengel A. Mean platelet volume in patients with metabolic syndrome and its relationship with coronary artery disease Thromb Res. 2007; 120: 245-50.

37. Dursunoglu D, Evrengül H, Tanriverdi H, Kuru Ö, Gür F, Kaftan A, et al. Do female patients with metabolic syndrome have masked left ventricular dysfunction? Anadolu Kardiyol Derg. 2005; 5: 283-8.

38. Gorter PM, Olijhoek JK, Graaf YVD, Algra A, Rabelink TJ, Visseren FLJ. Prevalence of the metabolic syndrome in patients with coronary heart disease, cerebrovascular disease, peripheral arterial disease or abdominal 
aortic aneurysm. Atherosclerosis. 2004; 173: 363-9.

39. Reilly M, Wolfe ML, Rhodes T, Girman C, Mehta N, Rader DJ. Measures of insulin resistance add incremental value to the clinical diagnosis of metabolic syndrome in association with coronary atherosclerosis. Circulation. 2004; 110: 803-9.

40. Scuteri A, Najjar SS, Muller DC, Andres R, Hougaku H, Metter EJ, et al. Metabolic syndrome amplifies the age-associated increases in vascular thickness and stiffness. J Am Coll Cardiol. 2004; 43: 1388-95.

41. Noto D, Barbagallo CM, Cefalú AB, Falletta A, Sapienza M, Cavera G, et al. The metabolic syndrome predicts cardiovascular events in subjects with normal fasting glucose: results of a 15 years follow-up in a mediterranean population. Atherosclerosis. 2008; 197: 147-53.

42. Empana JP, Zureik M, GariepyJ, Courbon D, Dartigues JF, Ritchie K, et al. The metabolic syndrome and the carotid artery structure in noninstitutionalized elderly subjects the three-city study. Stroke. 2007; 38: 893-9.

43. Kurl S, Laukkanen JA, Niskanen L, Laaksonen D, Sivenius J, Nyyssonen K, et al. Metabolic syndrome and the risk of stroke in middle-aged men. Stroke. 2006; 37: 806-11.

44. Langenberg C, Bergstrom J, Scheidt-Nave C, Pfeilschifter J, Barret-Connor E. Cardiovascular death and the metabolic syndrome role of adipositysignaling hormones and inflammatory markers. Diabetes Care. 2006; 29: 1363-9.

45. Najarian RM, Sullivan LM, Kannel WB, Wilson PWF, D'Agostino RB, Wolf PA. Metabolic syndrome compared with type 2 Diabetes Mellitus as a risk factor for stroke: the Framingham Offspring Study. Arch Intern Med. 2006; 166: 106-11.

46. Iglseder B, Cip P, Malaimare L, Ladurner G, Paulweber B. The metabolic syndrome is a stronger risk factor for early carotid atherosclerosis in women than in men. Stroke. $2005 ; 36: 1212-7$.

47. Malik S, Wong ND, Franklin SS, Kamath TV, L'Italien GJ, Pio JR, et al. Impact of the metabolic syndrome on mortality from coronary heart disease, cardiovascular disease, and all causes in United States adults. Circulation. 2004; 110: 1245-50
48. Marroquin OC, Kip KE, Kelley DE, Johnson BD, Shaw LJ, Merz CNB, et al. Metabolic syndrome modifies the cardiovascular risk associated with angiographic coronary artery disease in women: a report from the women's ischemia syndrome evaluation. Circulation. 2004; 109: 714-21.

49. Bellia C, Bivona G, Scazzone C, Ciaccio M. Association between homocysteinemia and metabolic syndrome in patients with cardiovascular disease. Ther Clin Risk Manag. 2007; 3 (6): 999-1001.

50. Montalcini T, Gorgone G, Federico D, Ceravolo R, Emanuele V, Sesti G, et al. Association of LDL cholesterol with carotid atherosclerosis in menopausa women affected by the metabolic syndrome. Nutr Metab Cardiovasc Dis. 2005; $15: 368-72$

51. Wang J, Ruotsalainen S, Moilanen L, Lepisto, P, Laakso M, Kuusisto J. The metabolic syndrome predicts cardiovascular mortality: a 13-year follow-up study in elderly non-diabetic Finns. Eur Heart J. 2007; 28: 857-64.

52. Zeller M, Steg PG, Ravisy J, Laurent Y, Janin-Manificat L, L'Huillier I, et al. Prevalence and impact of metabolic syndrome on hospital outcomes in acute myocardial infarction. Arch Intern Med. 2005; 165: 1192-8.

53. Holvoet P, Lee DH, Steffes M, Gross M, Jacobs DR Jr. Association between circulating oxidized low-density lipoprotein and incidence of the metabolic syndrome. JAMA. 2008; 299 (19): 2287-93.

54. Skoumas J, Papadimitriou L, Pitsavos C, Masoura C, Giotsas N, Chrysohoou C, et al. Metabolic syndrome prevalence and characteristics in Greek adults with familial combined hyperlipidemia. Metabolism. 2007; 56: 135-41.

55. Iribarren C, Go AS, Husson G, Sidney S, Fair JM, Quertermous T, et al Metabolic syndrome and early-onset coronary artery disease. Is the whole greater than its parts?. J Am Coll Cardiol. 2006; 48: 1800-7.

56. Meigs JB, Wilson PWF, Fox CS, Vasan RS, Nathan DM, Sullivan LM, et al. Body Mass Index, Metabolic Syndrome, and Risk of Type 2 Diabetes or Cardiovascular Disease. J Clin Endocrinol Metab. 2006; 91: 2906-12.

57. Sociedade Brasileira de Cardiologia. I Diretriz brasileira de diagnóstico e tratamento da síndrome metabólica. Arq Bras Cardiol. 2005; 84 (1): 15-24.

58. Sociedade Brasileira de Cardiologia. IV Diretriz brasileira sobre dislipidemias e prevenção da aterosclerose. Arq Bras Cardiol. 2007; 88 (1): 2-19. 